\title{
A new approach to $q$-linear Diophantine fuzzy emergency decision support system for COVID19
}

\author{
Alaa O. Almagrabi ${ }^{1}$-Saleem Abdullah ${ }^{3} \cdot$ Maria Shams $^{3}$ (i) $\cdot$ Yasser D. Al-Otaibi ${ }^{2} \cdot$ Shahzaib Ashraf $^{3}$
}

Received: 27 July 2020 / Accepted: 9 March 2021 / Published online: 5 April 2021

(C) The Author(s), under exclusive licence to Springer-Verlag GmbH Germany, part of Springer Nature 2021

\begin{abstract}
The emergency situation of COVID-19 is a very important problem for emergency decision support systems. Control of the spread of COVID-19 in emergency situations across the world is a challenge and therefore the aim of this study is to propose a q-linear Diophantine fuzzy decision-making model for the control and diagnose COVID19. Basically, the paper includes three main parts for the achievement of appropriate and accurate measures to address the situation of emergency decision-making. First, we propose a novel generalization of Pythagorean fuzzy set, q-rung orthopair fuzzy set and linear Diophantine fuzzy set, called q-linear Diophantine fuzzy set (q-LDFS) and also discussed their important properties. In addition, aggregation operators play an effective role in aggregating uncertainty in decision-making problems. Therefore, algebraic norms based on certain operating laws for q-LDFSs are established. In the second part of the paper, we propose series of averaging and geometric aggregation operators based on defined operating laws under q-LDFS. The final part of the paper consists of two ranking algorithms based on proposed aggregation operators to address the emergency situation of COVID-19 under q-linear Diophantine fuzzy information. In addition, the numerical case study of the novel carnivorous (COVID-19) situation is provided as an application for emergency decision-making based on the proposed algorithms. Results explore the effectiveness of our proposed methodologies and provide accurate emergency measures to address the global uncertainty of COVID-19.
\end{abstract}

Keywords q-Linear Diophantine fuzzy informations · q-Linear Diophantine fuzzy set · Aggregation information . Emergency decision support systems · COVID19

Maria Shams

mariaakhunzada@gmail.com

Alaa O. Almagrabi

aalmagrabi3@kau.edu.sa

Saleem Abdullah

saleemabdullah81@yahoo.com

Yasser D. Al-Otaibi

yalotaibi@kau.edu.sa

Shahzaib Ashraf

shahzaibashraf@awkum.edu.pk

1 Department of Information Systems, Faculty of Computing and Information Technology, King Abdulaziz University, Jeddah 21589, Saudi Arabia

2 Department of Information Systems, Faculty of Computing and Information Technology in Rabigh, King Abdulaziz University, Jeddah 21589, Saudi Arabia

3 Department of Mathematics, Abdul Wali Khan University Mardan, Mardan, Khyber Pakhtunkhwa, Pakistan

\section{Introduction}

The human beings faced different challenges during the rapid cycle of the 21 st century, such 9/11 terrorist attacks in 2001, the Catalina hurricane in 2005, the Pakistan earthquake in 2005, Wenchuan eartquack explosion accident at Port Group in Tianjin, 2015. Now the current epidemic of corona virus disease. The spreading of the corona virus in the world is very quickly and the 200 countries effected from corona virus. The first case of corona virus reported in 2019 in Wuhan, capital of Hubei province, China. In December, 2019 , some cases were reported of the same symptoms and then diagnose a new type of corona virus (nCoViD19), later the World Health Organization (WHO), rename the corona virus as COVID-19. In current situation throughout the world many people suffer from the COVID-19 epidemic. The life of people on earth is very difficult in current situation due to the lookdown. The WHO declared health emergency situation and also called COVID-19 as epidemic. In 
the emergency situation, the decision-makers (DMs) or the crisis response department should develop policies or use an efficient emergency solution to avoid any more worsening of the condition. Taking fast and fair decision the emergency is a concern for the field of emerging management. Emergency decision-making has been a necessary aspect of emergency services in many countries and a subject of study in educational contexts. In this case, when making decisions (Liu et al. 2018a; Ren et al. 2017; Wang et al. 2015), people are generally restricted logically rather than absolutely logical. It is therefore important to establish decision-making approaches that understand human behaviors in order to provide efficient ways for COVID-19 people to respond in emergency situations.

Risk decision method: Several approaches for evaluating decisions have been proposed to solve emergency rescue problems (Hämäläinen et al. 2000; Yu and Lai 2011). In future reference, (Hämäläinen et al. 2000) suggested a technique of selecting a suitable response action to protect the population in a nuclear accident based on multiple attributes utility theory (MAUT). Such research findings included different methods of decision analysis to help the decisionmaking of the DMs for emergency response. However, the DM's performance is usually ignored to be a risk decision analysis (RDA) method for emergency management. Several psychological experiments demonstrated that there are some risky and uncertain psychological features of human behavior, such as reference dependency, loss aversion and judgmental distortion of the probability of near impossible and definite outcomes (Kahneman and Tversky 1979; Schmidt et al. 2008; Quiggin 1991). Hence, it is necessary to examine risk decision analysis approaches that recognize human actions in order to provide effective decision support to the DM in emergency response (i.e. included, disease detection, awareness creation, use of protective clothing, face mask etc.). Creating online first-aid courses which will aware people to know about their duties and responsibilities during COVID-19 (Newey et al. 2020). (Ashraf et al. 2020a) introduced the novel emergency decision making using spherical intelligent fuzzy decision process to diagnose of COVID19. (Liu et al. 2014) have proposed a Risk Decision (RD) model based on the cumulative prospect theory (CPT) to address the problem of RD making in an emergency situations. (Ashraf and Abdullah 2020) presented the Emergency decision support modeling for COVID-19 under spherical fuzzy information.

Interval dynamic reference point method (IDRPM): The recent outbreak of COVID-19 has had a serious negative impact on human community and economic growth. In the event of such a devastating accident, it is of practical importance whether to take effective and suitable steps to monitor the worsening and deterioration of the situation (i.e. clinical management, first aid training, improved personal protective equipment, restricted intercity transport etc.). As a result, an essential research discussion for emergency management is how to respond in a timely and effective manner, or how to choose a satisfying alternative at the initial stages of an emergency, and many researchers have discussed such issues. (Wang et al. 2015) presented an IDRPM, focusing mainly on inadequate or incomplete information in emergency situations, and choosing optimal emergency alternatives, while ignoring the psychological behavior of DMs in emergency situations.

The conditions for risk aversion: Aversion to the spread of mean preservation implies aversion to the risk. Aversion to mean-preserving spread, sometimes referred to as strong risk aversion, is more restrictive than risk aversion (often referred to as weak risk aversion) for general decision models. The prevention and control of Corona virus and also to overcome the spreading of this disease we should implies risk aversion according to the condition The propose novel offers a number of solution and risk aversion for the prevention and control of COVID-19. Here we present some strong risk aversion on the basis of which people become safe and prevent from the effect of COVID-19 such as vaccination, medical support is mandatory, Creating online firstaid courses, isolate the affected or suspected person from other healthy people and Trained Technician etc. Through cumulative prospect theory, Schmidt and Zank defined the conditions for risk aversion. In (Liu et al. 2011) put forward a technique to risk decision making issues with likelihood of intervals based on the prospect principle.

As the social disruptions from COVID-19 is spreading in the world, and questions arises for many about the role of decision maker's and fuzzy logic. Because decision maker's and fuzzy logic have its importance to handle such pentamic situations. Every organization follow three steps (given in Fig. 1) for the solution of any problem which are; planning, implementing and then taking decision.

Intuitionistic fuzzy set theory: Problems related to uncertain situations occur frequently in DM, but are demanding due to the difficult modeling and controlling situation of these uncertainties. Atanassov (1986) suggested the idea of intuitionistic fuzzy set (IFS) as an extension of FS (Zadeh 1965) by adding the terms of non-membership grades (NMG) to membership grades (MG) with the condition that addition of MG and NMG bounded with unity. Geometric representation of IF objects was given by (Atanassov 1989). Later it expanded to the interval-valued IFS (IVIFS) (Atanassov and Gargov 1989), categorized by an interval valued MG and NMG. For IFNs, Xu and Yager (2006) introduced certain weighted geometric aggregation operators. (Garg 2016a) adopted the t-norm operations of Einstein for IFS. Besides these, the researchers (Xu 2005; Wang et al. 2009; Khan et al. 2019a, 2020a, b, c; Herrera and Martínez 2001; Garg 2016b) have attracted much attention 


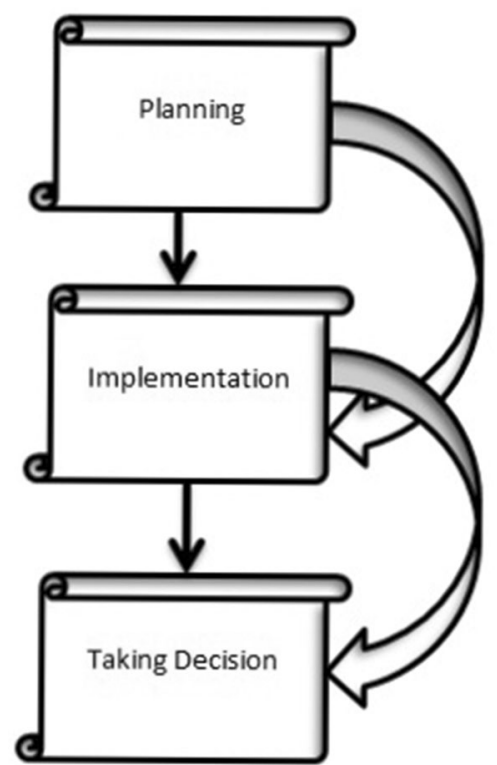

Fig. 1 Three-steps solution

from the IFS and IVIFS over the past three decades. These studies can deal the uncertain information that defined in quantitative aspects. (Zhang 2014) defined the linguistic intuitionistic fuzzy set (LIFS). The IFS failed due to constraint the space for MG and NMG, so Yager developed new approach called Pythagorean fuzzy set (PyFS).

Pythagorean fuzzy sets: Yager (2013a, 2013b) introduced the Pythagorean fuzzy set (PyFS), which is an extended version of IFS concept and satisfies the restrictions that the square sum of its MG and NMG is less than or equal to one. For instance, (Peng and Garg 2019) studied the Multiparametric similarity measures on PyFSs. Furthermore, (Yager 2013b) introduced some aggregation operators (AOs) in the PyFS environment, while (Garg 2019a) extented PyFSs to new logarithmic operational laws and their aggregation operators. (Khan et al. 2019b) established the Pythagorean fuzzy Dombi aggregation operators and discuused their application in decision making. (Ashraf et al. 2020b) presented the fuzzy decision support modeling for internet finance soft power evaluation based on sine trigonometric Pythagorean fuzzy information. (Batool et al. 2020) presented the novel concept of Pythagorean probabilistic hesitant fuzzy set and disccused their applicability in decision making. (Garg 2016c, 2017) introduced operations of Einstein t-norm for PyFNs. (Ma and Xu 2016) built some symmetric PyF AOs. The ideas of PyF interaction power Bonferroni mean aggregation operators in MADM were investigated by (Wang and Li 2020). (Zeng 2017) provided the details on probabilistic and ordered weighted averaging (OWA). Garg (2018a) suggested some strategic DM methods for solving MCDM problems with immediate probabilities under the PyF framework. Garg (2018b) recently implemented a Linguistic PyFS and also proposed a linear programming model-based solution for DMP (Garg 2018c).

q-rung orthopair fuzzy set: Few researchers have investigated another concept called q-rung orthopair fuzzy set (q-ROFS) (Liu and Wang 2018a; Ali 2018) to expand the space of IFS and PyFS. Although IFSs or PyFSs are widely studied and applied in different fields, their scope for providing information remains restricted. Recently, to resolve it, the q-ROFSs, initially developed by (Yager 2016), is a more effective method for defining data vagueness than the IFSs and PyFSs. The q-ROFSs are also defined as twograde knowledge like $\wp$ and $\Re$, with qth power restriction i.e. $0 \leq \wp^{q}+\mathfrak{R}^{q} \leq 1, q \geq 1$. It is clearly understood that q-ROFSs is more specific than IFS and PyFS and the respective set reduces to IFSs and PyFSs by setting $q=1$ and $q=2$. q-ROFSs can be classified into three factors:

1) The basic operational laws are the first and the main factor. Du (2019a) showed the arithmetic operations in relation to generalized OFSs. (Gao et al. 2018) outlined the idea of the continuities and differentials of q-ROF functions. Ye et al. (2019) presented for q-ROFSs. (Peng et al. 2018) described for q-ROFNs.

2) The second factor is the AOs which are an efficient tool using DMP. Liu and Wang (2018a) presented. Liu et al. (2018b) outlined. (Xing et al. 2019) described for q-ROFSs. Many other categories of q-ROFNs were described, such as the weighted Bonferroni mean (WBM) by (Liu 2018), the weighted Archimedean BM (Liu and Wang 2018b), the weighted Heronian mean (HM) (Wei et al. 2018), the weighted partitioned HM (Liu et al. 2018c), the exponential and logarithm (Peng et al. 2018), the weighted Maclaurin symmetric mean (MSM) (Wei et al. 2019), the weighted power partitioned MSM (Bai et al. 2018) and the weighted point operators (Xing et al. 2019) for aggregating the DM information given by experts.

3) The third factor of the decision maker is to rank the alternatives. For it, many researchers have used the traditional methods such as correlation and correlation coeffcient (Du 2019b), distance measures (Peng and Dai 2019), similarity measures (Wang et al. 2019), application of qROFSs in practical MAGDM problems (Wang and Li 2018), are gaining importance and popularity within academia under the q-ROFSs for solving the MAGDM.

Gou and Xu (2017) defined the exponential operational laws (EOLs) for IFSs. However, in terms of PyFSs, Garg presented (Garg 2018d, 2019b).

Linear Diophantine fuzzy set: IFSs, PyFSs, and q-ROFSs ideas have wide range of applications in different real-life fields, however these concepts use their own limitations relevent to MG and NMG. Riaz and Hashmi (2019) introduced the novel idea of Linear Diophantine Fuzzy Set (LDFS) with the introduction of reference parameters 
(RPs) to remove these limitations. Because of the use of RPs, LDFS model is more effective and versatile than other approaches. This collection filled the spaces of existing systems, and with addition of RPs extended the space for MG and NMG. LDFSs also presented two grades of information, the sum of which is bounded by one, and its parameters sum is also bounded by one, such as the sum of product of reference parameters with MG and NMG respectively is less than or equal to 1 .

So here again, under certain real world problem, the sum of the reference parameters that an alternative fulfil the attribute obtained by DM is often larger than one, so LDFS did not achieved his goal related to reference parameters. The LDFS have their own limitations related to the reference parameters. Therefore, we introduce the novel concept of the q-rung LDFS (q-RLDFS). In q-RLDFS we introduce the qth power of reference parameter which cover the space of existing structure and cover the space of MG and NMG with the help of qth power of reference parameters. In the entire manuscript, the motivation for the proposed model is described step by step. Now we're addressing some main goals of this article.

(1) Under certain real world problem, the sum of MG and NMG in any types of FS is sometimes greater than 1 for example $0.8+0.7>1$ and the square sum may also be greater than 1 (e.g $0.8^{2}+0.7^{2}>1$ ). In such cases, IFS and PyFS have failed. In the case of q-ROFS, the conditions on MG and NMG are modified to $0 \leq \wp^{q}+\Re^{q} \leq 1$ in order to overcome these deficiencies. We can handle MG and NMG even for extremely large values of $\}\} q^{\prime \prime}$. In certain practical issues we obtain $1^{q}+1^{q}>1$, which violates the restriction of q-ROFS, if both MG $\wp$ and NMG $\Re$ are equal to 1 (i.e. $\wp=\Re=1$ ). In ordered to remove such restriction, (Riaz and Hashmi 2019) introduced concept of LDFS in which they introduced the role of reference parameters, which hold the condition $0 \leq(\alpha) \wp_{D(\hbar)}+(\beta) \Re_{D(\hbar)} \leq 1 \forall \hbar \in M$ with $0 \leq \alpha+\beta \leq 1$. But here again, the sum of reference parameters provided by DM may lager than one i.e. $\alpha+\beta>1$, which contradicts the constraint of LDFS. So LDFS did not achieved his goal related to reference parameters. It makes the MADM limited, and affects the optimum decision. In order to eliminate this contradiction, we introduce the novel idea of the q-RLDFS which is capable of dealing with these situations. To explain the concept of q-RLDFSs, we have three objectives related to our proposed method;

- Our first goal is to fill this knowledge gap with the new q-RLDFS approach with $q \geq 1$. Through this approach, under the influence of reference parameters, we can solve the IF, PyF, q-ROF, LDF structure (e.g for $(0.8+0.7>1)$, entrance of RPs and by setting $q=1$ such that $(0.8)(0.7)^{1}+(0.7)(0.6)^{1}<1$, where $\langle 0.7,0.6\rangle$ can be used as a couple of RPs, respectively, for MG and NMG. As this suggested framework's comparable to a very well-known $\operatorname{LDE}(a) x+(b) y=c$ in the number theory and the adding of qth power of reference parameter makes it look like q-RLDFS is the most appropriate name for the developed framework.

- The second objective is to implement the function of qth power of reference parameters (RPs) in q-RLDFS while IFSs, PyFSs, q-ROFSs and LDFSs can not handle qth parameters. The suggested framework improves existing methodologies and the decision maker can choose the grades freely without any restrictions. This framework also describes the problem by changing the physical sense of reference. The respective collection reduces to LDFS by setting $q=1$, respectively. Furthermore, it should be noted that the Diophantine space increases as we increase the rung $q$ and therefore the boundary limits have a greater search space which can convey a broader variety of the fuzzy data. Therefore, we can express a wider range of fuzzy information by using q- RLDFSs. In other words, we can continue to adjust the value of the parameter $q$ to determine the information expression range, and thus q-RLDFSs are more flexible and more suitable for the uncertain environment.

- Our third aim is to build close relationships between the present study and problems with the MADM. We established two major algorithms to handle the data in a parametric manner with multi-attribute complexities. Interestingly, both algorithms yield to the same result.

The layout of this paper is organized as; Sect. 2 provides some basic concepts of FS, IFS, PFS, q- ROFS and LDFS. In Sect. 3, we introduce the novel concept of q-RLDFS, and develop certain q-RLDFS operations using the illustrations. Section 4, introduces the idea of q-RLDFSs for the concept of q-RLDFWA, q-RLDFOWA and q-RLDFHWA operators . Section 5 introduces the idea of q-RLDFSs for the definition of q-RLDFWG, q-RLDFOWG and q-RLDFHWG operators, and also offers different score and accuracy for comparing q-RLDFNs with different orders. Section 6, presents the concept of MADM with the support of q-RLDFWA and q-RLDFWG aggregation operators. A case study on Coronavirus disease 2019 (COVID-19) in Wuhan, china, in December 2019 is given to illustrate the application of the proposed method by using two different algorithms under q-RLDF environment and its associated score function that how to reduce the disease its preventive alternative is establish in Sect. 7. In Sect. 8, we introduce a detailed comparison between the proposed method and existing methods and see the aggregated results as the influence of score functions on the final selection. The end of this work is eventually outlined in Sect. 9. 


\section{Basic concepts}

In this section, we introduce some elementary definitions of fuzzy set and intuitionistic fuzzy set etc. In order to develop a new cocept, first we review the basic concept and properties for understanding the concept.

Definition 1 Zadeh (1965) Suppose an arbitrary nonempty set $M$. A fuzzy set (FS) $L$ is defined on $M$ as;

$L=\left\{\left(\hbar, \wp_{L}(\hbar)\right) \mid \hbar \in M\right\}$

Here the function $\wp_{L}$ is a transformation of $M$ to $[0,1]$, and for every $\hbar \in M, 0 \leq \wp_{L}(\hbar) \leq 1$, and function $\wp_{L}(\hbar)$ are said to be the MG of $\hbar$ in $M$.

Atanassov give the idea of positive membership and negative membership function with the restriction that addition of both function is bounded by one.

Definition 2 (Atanassov 1986) Consider a fixed set $M$ and an IFS $A$ in $M$ is defined as;

$A=\left\{\left(\hbar, \wp_{A(\hbar)}, \mathfrak{R}_{A(\hbar)}\right) \mid \hbar \in M\right\}$

where $\wp_{A}$ and $\mathfrak{R}_{A} \in[0,1]$ are called $\mathrm{MG}$ and NMG functions, respectively and with such that $\forall \hbar \in M$, $0 \leq \wp_{A(\hbar)}+\Re_{A(\hbar)} \leq 1$.

Sometime in real life problems, IFS cannot deal when $\wp_{A(\hbar)}+\mathfrak{R}_{A(\hbar)}>1$. To solve this drawback Yager extend IFS concept named as PFS.

Definition 3 (Yager 2013a, b) Consider a fixed set $M$ and the PyFS is denoted by $A_{P}$ and mathematical defined as

$A_{P}=\left\{\left(\hbar, \wp_{A P(\hbar)}, \mathfrak{R}_{A P(\hbar)}\right) \mid \hbar \in M\right\}$,

where $\wp_{A P(\hbar)}$ and $\Re_{A P(\hbar)} \in[0,1]$ are MG and NMG functions with subject to $\left(\wp_{A P(\hbar)}\right)^{2}+\left(\Re_{A P(\hbar)}\right)^{2} \leq 1$. The hesitancy MG is denoted by

$\pi_{A P(\hbar)}=\sqrt{1-\left(\wp_{A P(\hbar)}\right)^{2}-\left(\Re_{A P(\hbar)}\right)^{2}}$.

The IFS and PFS fail in situation when sum of MG and NMG and the sum of squares is also larger than one, hence Yager (2016) launched a generalization of IFS and PFS, so called q-ROFS.

Definition 4 (Yager 2016) Suppose $M$ be a fixed set. A q-ROFS $B$ on $M$ have the following mathematical symbol;

$B=\left\{\left(\hbar, \wp_{q(\hbar)}, \Re_{q(\hbar)}\right): \hbar \in M\right\}$ where $\wp_{q(\hbar)}$ and $\Re_{q(\hbar)} \in[0,1]$ are MG and NMG functions with subject to $0 \leq\left(\wp_{q(\hbar)}\right)^{q}+\left(\mathfrak{R}_{q(\hbar)}\right)^{q} \leq 1 ; q \geq 1$. The hesitancy part is denoted as

$\pi_{q(\hbar)}=\sqrt[q]{1-\left(\wp_{q(\hbar)}\right)^{q}-\left(\mathfrak{R}_{q(\hbar)}\right)^{q}}$

The q-ROFS has also certain restrictions on MG and NMGs. In order to remove these restrictions, Riaz and Hashmi (2019) implemented the theory of LDFS in which they added the structure of reference parameters (RPs) while IFS, PyFS and q-ROFS can not handle these reference parameters (RPs).

Definition 5 (Riaz and Hashmi 2019) Suppose $M$ be a fixed non-empty reference set and the LDFS is denoted by $G_{D}$ and mathematical defined as:

$G_{D}=\left\{\left(\hbar,\left\langle\wp_{D(\hbar)}, \Re_{D(\hbar)}\right\rangle,\langle\alpha, \beta\rangle\right): \hbar \in M\right\}$

where $\wp_{D(\hbar)}, \mathfrak{R}_{D(\hbar)}, \alpha, \beta \in[0,1]$ are $\mathrm{MG}, \quad \mathrm{NMG}$ and RPs respectively, and hold the condition $0 \leq \alpha \wp_{D(\hbar)}+\beta \Re_{D(\hbar)} \leq 1, \quad \forall \hbar \in M$ with $0 \leq \alpha+\beta \leq 1$. Such reference parameters may help to describe or identify a specific model. Indeterminacy degree can be defined as;

$\Gamma \pi_{D}=1-(\alpha) \wp_{D(\hbar)}-(\beta) \Re_{D(\hbar)}$,

where $\Gamma$ is the reference parameter of the indeterminacy degree.

\section{Concept Of $q$-rung linear Diophantine fuzzy set ( $q$-RLDFS)}

But again, in certain real world problem, the addition of reference parameters of which an alternative satisfies DM's attribute might be lager than one, so LDFS has failed to achieved his goal related to reference parameters. To eradicate this contradiction, we present the novel concept of q-rung linear Diophantine fuzzy set (q-RLDFS) which has the capacity to solve with circumstances of this kind.

Definition 6 Suppose $M$ be a fixed non-empty reference set and the q-rung linear Diophantine fuzzy set (q-LDFS) is denoted by $T_{D q}$ and mathematical defined as:

$T_{D q}=\left\{\left(\hbar,\left\langle\wp_{D q(\hbar)}, \Re_{D q(\hbar)}\right\rangle,\langle\alpha, \beta\rangle\right): \hbar \in M\right\}$

where $\wp_{D q(\hbar)}, \mathfrak{R}_{D q(\hbar)}, \alpha, \beta \in[0,1]$ are MG, NMG and reference parameters (RPs) respectively. These functions fulfill the restriction; 
$0 \leq(\alpha)^{q} \wp_{D q(\hbar)}+(\beta)^{q} \Re_{D q(\hbar)} \leq 1 \forall \hbar \in M, q \geqslant 1$,

with $0 \leq \alpha^{q}+\beta^{q} \leq 1$. Such reference parameters may help to describe or identify a specific model. The part of the hesitation may be calculated as;

$\Gamma \pi_{D}=\sqrt[q]{1-\left((\alpha)^{q} \wp_{D q(\hbar)}+(\beta)^{q} \Re_{D q(\hbar)}\right)}$,

where $\Gamma$ represent the reference parameters associated with the degree of hesitation or indeterminacy. A particular system is categorize and defined by the reference parameters and these reference parameters also change the physical meaning (sense) of the system. They increasing the grade space used in q-RLDFS, and eliminate restrictions against them. In q-RLDFS we generalized the concept LDFS, in which we extend the reference parameter and categorizes as; the sum of the qth power of reference parameters is bounded by one, with addition of the qth power of RPs with MG and NMG respectively which fulfill the lack in reference parameters (RPs). This structure describes the problem by assigning various types of reference parameters $(\alpha, \beta)$. In our new presented concept i.e. (q-RLDFS) addition of qth power of reference parameters solve the deficiencies of LDFS. The proposed method of q-RLDFS is more efficient and flexible rather than other approaches due to the addition of qth power of reference parameters. This method construct strong relation with multi-attribute decision making (MADM) problems.

Definition 7 A q-rung linear Diophantine fuzzy number (q-RLDFN) is a collection of

$\varrho=\left\{\left\langle\wp_{D q}, \mathfrak{R}_{D q}\right\rangle,\langle\alpha, \beta\rangle\right\}$

where $\varrho$ represent the q-rung linear Diophantine fuzzy number with conditions;

(i) $0 \leq(\alpha)^{q}+(\beta)^{q} \leq 1, q \geqslant 1$,

(ii) $0 \leq(\alpha)^{q} \wp_{D q(\hbar)}+(\beta)^{q} \Re_{D q(\hbar)} \leq 1$

(iii) $0 \leq(\alpha), \wp_{D q(\hbar)},(\beta), \Re_{D q(\hbar)} \leq 1$

Next definition is about absolute q-rung linear Diophantine fuzzy set (absolute q-RLDFS) and null or empty q-rung linear Diophantine fuzzy set (q-RLDFS).

Definition 8 A q-RLDFS on $M$ of the form

${ }^{1} T_{D q}=\{(\hbar,(1,0),(1,0)): \hbar \in M\}$

is called absolute q-RLDFS and

${ }^{0} T_{D q}=\{(\hbar,(0,1),(0,1)): \hbar \in M\}$ is called empty or null q-RLDFS.

We know that, if

- We put $q=1$ in Definition 6, then q-RLDFS reduce to LDFS.

- We put $q=2$ in Definition 6, then q-RLDFS reduce to quadratic DFS.

- We put $q=3$ in Definition 6, then q-RLDFS reduce to cubic DFS.

- We put $q=4$ in Definition 6, then q-RLDFS reduce to bi-quadratic DFS and so on as shown in Fig.2.

Which are the advantages of q-LDFS for variations of values of q. It should be noted that the Diophantine space increases as we increase the rung $\mathrm{q}$ and therefore the boundary limits have a greater search space which can convey a broader variety of the fuzzy data.

It is observed that Atanassov's intuitionistic fuzzy sets are q-ROFs by setting $q=1$ and Yager's PyFSs are q-ROFs by setting $q=2$; as shown in Fig.2:

(1) Any intuitionistic fuzzy set is a q-ROF for all $q \geq 1$.

(2) An intuitionistic fuzzy set is a PyFS.

(3) Any PyF subset is a q-ROF for $q \geq 2$.

The above remarks identifies that LDFS, q-ROFS, PyFS, IFS and FS are the special cases of q-RLDFS.

Corollary 1 Any intuitionistics fuzzy set is a LDFS and, Any LDFS is $q$-RLDFs for $q=1$. But the converse is not true.

Corollary 2 Any Pythagorean fuzzy set is a LDFS and, Any $L D F S$ is $q$-RLDFs for $q=1$. But the converse is not true.

Any intuitionistics fuzzy set is LDFS and any LDFS is q-RLDFS but the converse is not true as shown in Fig. 3.

\subsection{Personnel selection for software company}

There are many practical applications of q-RLDFS in the fields of engineering, artificial intelligence, medical and MADM. In this novel one can see a wide variety of these implementations. Assume a software company needs to employ an analyst for the project. The software company set a criteria for selecting a perfect system analyst with a wide variety of characteristics and a low cost which was based on the following characteristics such were; Self-confidence, mental steadiness, oral communication skills, personality traits and past experience. Suppose $M=\left\{\hbar_{1}, \hbar_{2}, \hbar_{3}, \hbar_{4}, \hbar_{5}\right\}$ be the set of some system analyst. First suppose reference parameters $(\alpha, \beta)$ for the structure of q-RLDFS. Let $\alpha=$ mental steadiness, oral communication skills, personality 


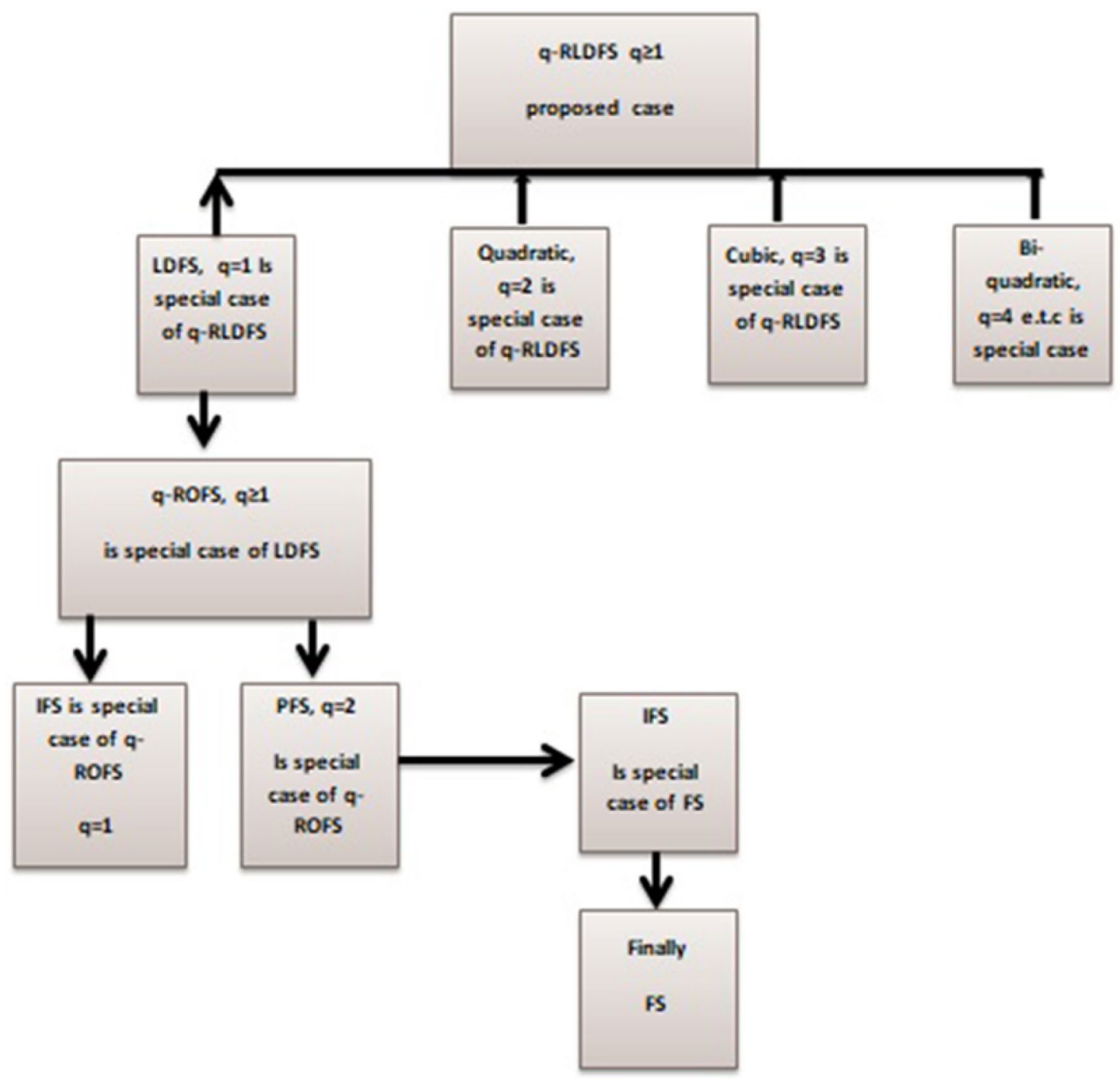

Fig. 2 Flow chart of $q$-RLDF concept

traits and $\beta=$ past experience and self-confidence. Table 1 represent the tabular form of q-RLDFS of such parameter for $q=3$. Now if we want the physical sense of such parameters to change, then data can be classified as q-RLDFS in other ways. For the second group, we can consider $\alpha=$ impress the company and $\beta=$ not impress the company. Table 2 represent the tabular form of q-RLDFS for the second group reference parameters.

Reference parameters have a major role to play. They describe some particular characteristics about criteria of company, like it is self-confidence, mental steadiness, oral communication skills, personality traits, past experience., qualified, not qualified etc, how much these characteristics were present in required one. The parameters values changes because of change in different criteria characteristics. The functions $\wp_{D q(\hbar)}, \mathfrak{R}_{D q(\hbar)}$ denote the required characteristics of software company, which shows that how many characteristics present in them, while reference parameters show that how much characteristics should be in them and $q \in \mathbb{N}$. The parameters are chosen by decision-maker's choice, whereas attribute grades are calculated from data collected. There are many advantages of reference parameters but one of the big advantage is that we are free to chose the attribute functions and are not bound by IFS, PFS, q-ROFS or LDFS conditions. For different set of parameters we can easily define different q-RLDFS on the same reference set $M$. Such parameters make our mathematical model more spatial.

\subsection{Buying of a laptop}

Think in this case a person interested in buying a laptop from the best brand, there are several brands for him to 


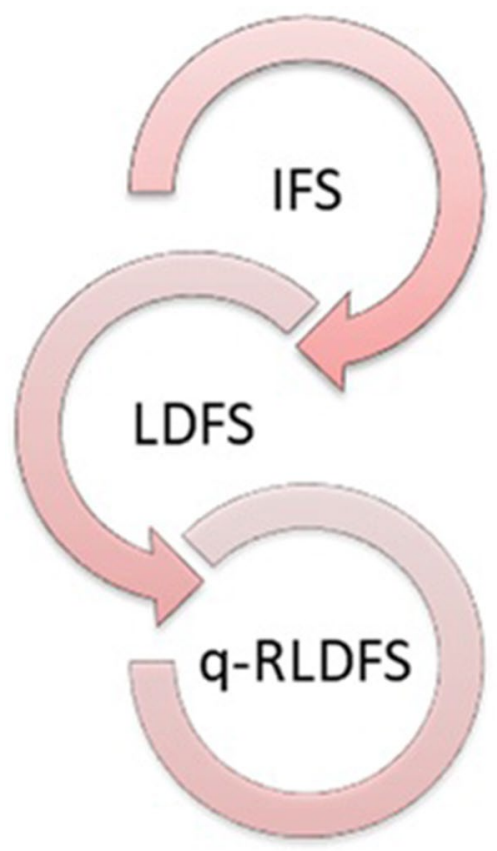

Fig. 3 Extension of IFS, PFS to $q$-RLDFS

Table 1 q-RLDFS (1st group)

\begin{tabular}{ll}
\hline$T_{D q}$ & $\left(\left\langle\wp_{D q(\hbar)}, \mathfrak{R}_{D q(\hbar)}\right\rangle,\langle\alpha, \beta\rangle\right), q=3$ \\
\hline$\hbar_{1}$ & $(\langle .9,1\rangle,\langle .9, .6\rangle)$ \\
$\hbar_{2}$ & $(\langle .82,1\rangle,\langle .8, .7\rangle)$ \\
$\hbar_{3}$ & $(\langle .95, .85\rangle,\langle .7, .8\rangle)$ \\
$\hbar_{4}$ & $(\langle .8,1\rangle,\langle .8, .7\rangle)$ \\
$\hbar_{5}$ & $(\langle .95, .9\rangle,\langle .8, .7\rangle)$ \\
\hline
\end{tabular}

Table 2 q-RLDFS (2nd group)

\begin{tabular}{ll}
\hline$T_{D q}$ & $\left(\left\langle\wp_{D q(\hbar)}, \Re_{D q(\hbar)}\right\rangle,\langle\alpha, \beta\rangle\right), q=3$ \\
\hline$\hbar_{1}$ & $(\langle 1,1\rangle,\langle .8, .7\rangle)$ \\
$\hbar_{2}$ & $(\langle .9, .87\rangle,\langle .6, .9\rangle)$ \\
$\hbar_{3}$ & $(\langle .85, .88\rangle,\langle .6, .9\rangle)$ \\
$\hbar_{4}$ & $(\langle .8,1\rangle,\langle .8, .7\rangle)$ \\
$\hbar_{5}$ & $(\langle .9, .87\rangle,\langle .6, .9\rangle)$ \\
\hline
\end{tabular}

choose, such as ThinkPad, Apple, Acer, HP, Haier and so forth. The person select a list of four brands for buying a laptop named as; ThinkPad, Apple, Acer and HP. He finds that it is hard for him to decide which one brand is the best. Let $M=\left\{\hbar_{1}, \hbar_{2}, \hbar_{3}, \hbar_{4},\right\}$ be an assembling of well known brands, where $\hbar_{1}=$ ThinkPad, $\hbar_{2}=$ Apple, $\hbar_{3}=$ Acer, $\hbar_{4}=$ HP.

So we can easily build the input information in the sense of q-RLDFSs, where MG ad NMG $\left\langle\wp_{D q}, \mathfrak{R}_{D q}\right\rangle$, indicates
Table 3 q-RLDFS (1st brand)

\begin{tabular}{ll}
\hline$T_{D q}$ & $\left(\left\langle\wp_{D q(\hbar)}, \mathfrak{R}_{D q(\hbar)}\right\rangle,\langle\alpha, \beta\rangle\right)$ \\
\hline$\hbar_{1}$ & $(\langle .8, .9\rangle,\langle .85, .7\rangle)$ \\
$\hbar_{2}$ & $(\langle .88, .95\rangle,\langle .8, .7\rangle)$ \\
$\hbar_{3}$ & $(\langle 1,1\rangle,\langle .8, .7\rangle)$ \\
$\hbar_{4}$ & $(\langle 1, .9\rangle,\langle .6, .9\rangle)$ \\
\hline
\end{tabular}

Table 4 q-RLDFS (2nd brand)

\begin{tabular}{ll}
\hline$T_{D q}$ & $\left(\left\langle\wp_{D q(\hbar)}, \mathfrak{R}_{D q(\hbar)}\right\rangle,\langle\alpha, \beta\rangle\right)$ \\
\hline$\hbar_{1}$ & $(\langle 1,1\rangle,\langle .7, .8\rangle)$ \\
$\hbar_{2}$ & $(\langle .99, .88\rangle,\langle .7, .85\rangle)$ \\
$\hbar_{3}$ & $(\langle .85, .88\rangle,\langle .6, .9\rangle)$ \\
$\hbar_{4}$ & $(\langle .9, .87\rangle,\langle .6, .9\rangle)$ \\
\hline
\end{tabular}

Table 5 q-RLDFS ( 3rd brand)

\begin{tabular}{ll}
\hline$T_{D q}$ & $\left(\left\langle\wp_{D q(\hbar)}, \Re_{D q(\hbar)}\right\rangle,\langle\alpha, \beta\rangle\right), q=3$ \\
\hline$\hbar_{1}$ & $(\langle .9,1\rangle,\langle .9, .6\rangle)$ \\
$\hbar_{2}$ & $(\langle 1, .9\rangle,\langle .7, .8\rangle)$ \\
$\hbar_{3}$ & $(\langle .9,1\rangle,\langle .9, .6\rangle)$ \\
$\hbar_{4}$ & $(\langle .87, .95\rangle,\langle .8, .7\rangle)$ \\
\hline
\end{tabular}

us about the brand's satisfaction and dissatisfaction and reference parameters $\langle\alpha, \beta\rangle$ represent the "extremely best software" and "not so best software" respectively. These parameters often refer the concept to the linguistic terms, and often refer to the attribute properties or qualities. Depending on our preference or the problem demand we could update the physical meaning of such reference parameters. If we change meaning of reference parameter then it become as; $\alpha=$ cheap and $\beta=$ expensive or $\alpha=$ consume less electricity and $\beta=$ consume more electricity, etc. The beauty of these qth power of parameters is that they improve MG and NMG space and increase the problem's variety of parameterization. Tables 3, 4 and 5 represent q-RLDFS to buy best brand laptop of such reference parameters.

Definition 9 Let $\rho_{\psi}=\left(\left\langle{ }^{\psi} \wp_{D q},{ }^{\psi} \mathfrak{R}_{D q}\right\rangle,\left\langle{ }^{\psi} \alpha,{ }^{\psi} \beta\right\rangle\right)$ for $\psi \in \Delta$ be an assmembling of q-RLDFNs on the reference set $M$ and the scalar $\lambda>0$ then the following properties are satisfied;

(1) $\varrho_{1}^{c}=\left(\left\langle{ }^{1} \Re_{D q},{ }^{1} \wp_{D q}\right\rangle,\left\langle{ }^{1} \beta,{ }^{1} \alpha\right\rangle\right)$

(2) $\varrho_{1}=\varrho_{2} \Longleftrightarrow{ }^{1} \wp_{D q}={ }^{2} \wp_{D q},{ }^{1} \mathfrak{R}_{D q}={ }^{2} \Re_{D q},{ }^{1} \alpha={ }^{2} \alpha,{ }^{1} \beta={ }^{2} \beta$

(3) $\rho_{1} \subseteq \rho_{2} \Longleftrightarrow{ }^{1} \wp_{D q} \leq^{2} \wp_{D q},{ }^{1} \Re_{D q} \geq^{2} \Re_{D q}{ }^{1} \alpha \leq^{2} \alpha,{ }^{1} \beta \geq^{2} \beta$

(4) $\bigcup_{\psi \in \Delta} \varrho_{\psi}=\left(\left(\sup _{\psi \in \Delta}^{\psi} \wp_{D q}, \inf _{\psi \in \Delta}^{\psi} \Re_{D q}\right),\left(\sup _{\psi \in \Delta}^{\psi} \alpha, \inf _{\psi \in \Delta}^{\psi} \beta\right)\right)$

(5) $\left.\bigcap_{\psi \in \Delta} \varrho_{\psi}=\left(\operatorname{(inf}_{\psi \in \Delta}^{\psi} \wp_{D q}, \sup _{\psi \in \Delta}^{\psi} \Re_{D q}\right),\left(\inf _{\psi \in \Delta}^{\psi} \alpha, \sup _{\psi \in \Delta}^{\psi} \beta\right)\right)$ 


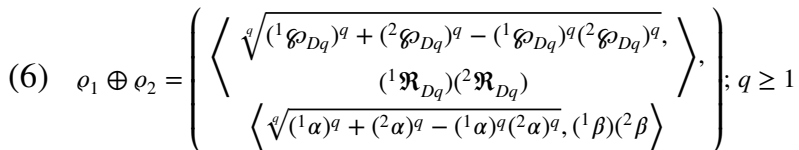

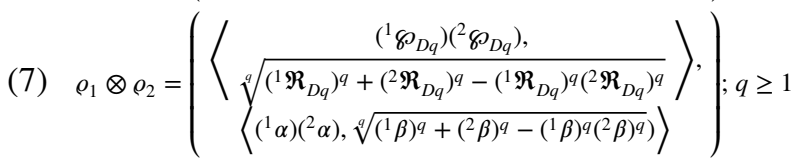

(8) $\lambda \varrho_{1}=\left(\begin{array}{c}\left\langle\sqrt[q]{1-\left(1-{ }^{1} \wp_{D q}^{q}\right)^{\lambda}},\left({ }^{1} \Re_{D q}\right)^{\lambda}\right\rangle \\ \left\langle\sqrt[q]{1-\left(1-{ }^{1} \alpha^{q}\right)^{\lambda}},{ }^{1} \beta^{\lambda}\right\rangle\end{array}\right) ; \lambda>0, q \geq 1$

(9) $\rho_{1}^{\lambda}=\left(\begin{array}{c}\left\langle\left({ }^{1} \wp_{D q}\right)^{\lambda}, \sqrt[q]{1-\left(1-{ }^{1} \mathfrak{R}_{D q}^{q}\right)^{\lambda}}\right\rangle \\ \left\langle 1 \alpha^{\lambda}, \sqrt[q]{1-\left(1-{ }^{1} \beta^{q}\right)^{\lambda}}\right\rangle\end{array}\right) ; \lambda>0, q \geq 1$.

Example 1 Let $\varrho_{1}=(\langle .8,1\rangle,\langle .8, .7\rangle)$ and $\varrho_{2}=(\langle .8, .9\rangle,\langle .85, .7\rangle)$ with $q=3$ be two q-RLDFNs then

(1) $\varrho_{1}^{c}=(\langle 1, .8\rangle,\langle .7, .8\rangle)$

(2) $\varrho_{1} \subseteq \varrho_{2}$, this property is also satisfy and clear from example by using above definition such that; $0.8=0.8,1>0.9,0.8<0.85,0.7=0.7$.

(3) $\varrho_{1} \cup \varrho_{2}=(\langle 0.8,0.9\rangle,\langle 0.85,0.7\rangle)=\varrho_{2}$

(4) $\varrho_{1} \cap \varrho_{2}=(\langle 0.8,1\rangle,\langle 0.8,0.7\rangle)=\varrho_{1}$

(5) $\varrho_{1} \oplus \varrho_{2}=(\langle 0.9134056,0.9\rangle,\langle 0.93288,0.49\rangle)$

(6) $\rho_{1} \otimes \varrho_{2}=(\langle 0.64,1\rangle,\langle 0.68,0.82849\rangle$

(7) $\lambda \varrho_{1}=(\langle 0.95969,1\rangle,\langle 0.95969,0.343\rangle), \lambda=3$

(8) $\varrho_{1}^{\lambda}=(\langle 0.512,1\rangle,\langle 0.512,0.89488\rangle), \lambda=3$.

Proposition 1 For two q-RLDFNs $\varrho_{1}$ and $\varrho_{2}$ with real numbers $\lambda>0$, then operation on these two q-RLDFNs are also $q-R L D F N s$ that is $\varrho_{1}^{c}, \varrho_{1} \cup \varrho_{2}, \varrho_{1} \cap \varrho_{2}, \varrho_{1} \oplus \varrho_{2}, \varrho_{1} \otimes \varrho_{2}, \lambda \varrho_{1}$ and $\rho_{1}^{\lambda}$ are also $q$-RLDFNs.

Proof This result can easily be proved by using of the above definition.

Proposition 2 Suppose we have three $q$-RLDFNs $\rho_{1}=\left(\left\langle{ }^{1} \wp_{D q},{ }^{1} \mathfrak{R}_{D q}\right\rangle,\left\langle{ }^{1} \alpha,{ }^{1} \beta\right\rangle\right), \rho_{2}=\left(\left\langle{ }^{2} \wp_{D q},{ }^{2} \Re_{D q}\right\rangle,\left\langle{ }^{2} \alpha,{ }^{2} \beta\right\rangle\right)$ and $\varrho_{3}=\left(\left\langle{ }^{3} \wp_{D q},{ }^{3} \Re_{D q}\right\rangle,\left\langle{ }^{3} \alpha,{ }^{3} \beta\right\rangle\right)$ then the following cases are satisfied;

(1) if $\varrho_{1} \subseteq \varrho_{2}$ and $\varrho_{2} \subseteq \varrho_{3}$ then $\varrho_{1} \subseteq \varrho_{3}$

(2) $\rho_{1} \cup \varrho_{2}=\rho_{2} \cup \varrho_{1}$

(3) $\rho_{1} \cap \varrho_{2}=\rho_{1} \cap \varrho_{2}$

(4) $\varrho_{1} \cup\left(\varrho_{2} \cup \varrho_{3}\right)=\left(\varrho_{1} \cup \varrho_{2}\right) \cup \varrho_{3}$

(5) $\varrho_{1} \cap\left(\varrho_{2} \cap \varrho_{3}\right)=\left(\varrho_{1} \cap \varrho_{2}\right) \cap \varrho_{3}$

(6) $\varrho_{1} \cup\left(\varrho_{2} \cap \varrho_{3}\right)=\left(\varrho_{1} \cup \varrho_{2}\right) \cap\left(\varrho_{1} \cup \varrho_{3}\right)$

(7) $\rho_{1} \cap\left(\varrho_{2} \cup \varrho_{3}\right)=\left(\varrho_{1} \cap \varrho_{2}\right) \cup\left(\varrho_{1} \cap \varrho_{3}\right)$

(8) $\left(\varrho_{1} \cup \varrho_{2}\right)^{c}=\varrho_{1}^{c} \cap \varrho_{2}^{c}$

(9) $\left(\varrho_{1} \cap \varrho_{2}\right)^{c}=\varrho_{1}^{c} \cup \varrho_{2}^{c}$

Proof Proof of the above statements are obvious.

\section{4 q-Rung linear Diophantine fuzzy weighted averaging aggregation ( $q$-RLDFWAA) operator}

In this section we define q-RLDFWA, q-RLDFOWA and q-RLDFHWA aggregation operators.

Definition 10 Let $\varrho_{D q \psi}=\left\{\left(\left\langle{ }^{\psi} \wp_{D q},{ }^{\psi} \mathfrak{R}_{D q}\right\rangle,\left\langle{ }^{\psi} \alpha,{ }^{\psi} \beta\right\rangle\right)\right.$ : $\psi=1,2, \ldots n\}$ be an assembling of q-RLDFNs on the reference set $M$ and the weight vector $\Omega=\left(\Omega_{1}, \Omega_{2}, \ldots, \Omega_{n}\right)^{T}$ with $\sum_{\psi=1}^{n} \Omega_{\psi}=1$, and $q \geq 1$; then the transformation $\theta: q-R \operatorname{LDFN}(M) \longrightarrow q-\operatorname{RLDFN}(M)$ is called q-rung linear Diophantine fuzzy weighted averaging aggregation operator (q-RLDFWAA) and defined as

$$
\begin{aligned}
q- & R L D F W A A\left(\varrho_{D q 1}, \varrho_{D q 2}, \varrho_{D q 3}, \ldots, \varrho_{D q n}\right) \\
& =\prod_{\psi=1}^{n}\left(\Omega_{\psi} \varrho_{D q \psi}\right) \\
& =\left(\left\langle\sqrt[q]{1-\prod_{\psi=1}^{n}\left(1-\psi \wp_{D q}^{q}\right)^{\Omega_{\psi}}}, \prod_{\psi=1}^{n} \psi \Re_{D q}^{\Omega_{\psi}}\right\rangle,\right), q \geq 1 .
\end{aligned}
$$

This operator can easily be proof by using the q-RLDFS operations and by mathematical induction. In q-RLDFWAA operator, $\varnothing$ denote positive membership and $\mathfrak{R}$ denote negative membership functions, $\alpha, \beta$ denote the reference parameters for both $\wp$ and $\Re$ respectively and $q \geq 1$. $\Omega$ denote the weights, $\varrho_{D q \psi}$ are the q-RLDFNs, where $\psi \in \mathbb{N}$ and q-RLDFN $(M)$ gathers all q-RLDFNs.

So, now let us we want to present q-RLDFOWAA operator.

Definition 11 Let $\varrho_{D q \psi}=\left\{\left(\left\langle{ }^{\psi} \wp_{D q},{ }^{\psi} \mathfrak{R}_{D q}\right\rangle,\left\langle{ }^{\psi} \alpha,{ }^{\psi} \beta\right\rangle\right)\right.$ : $\psi=1,2, \ldots n\}$ be an assembling of q-RLDFNs on the reference set $M$ and the weight vector $\Omega=\left(\Omega_{1}, \Omega_{2}, \ldots, \Omega_{n}\right)^{T}$ such that $\Omega>0$ with $\sum_{\psi=1}^{n} \Omega_{\psi}=1$, and $q \geq 1$; then the mapping $\theta: q-R \operatorname{LDFN}(M) \longrightarrow q-R \operatorname{LDFN}(M)$ is called q-rung linear Diophantine fuzzy ordered weighted averaging aggregation (q-RLDFOWAA) operator and defined as

$$
\begin{aligned}
& q-\operatorname{RLDFOWAA}\left(\varrho_{D q 1}, \varrho_{D q 2}, \varrho_{D q 3}, \ldots, \varrho_{D q n}\right) \\
& =\prod_{\psi=1}^{n}\left(\Omega_{\psi} \varrho_{D q \psi(\delta)}\right)
\end{aligned}
$$

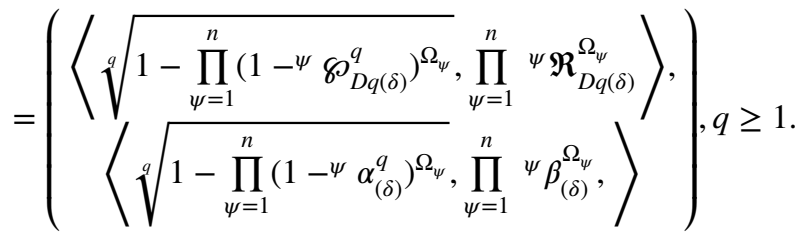


where $(\delta(1), \delta(2), \delta(3), \ldots, \delta(n))$ is the arrangement of $(\psi \in \mathbb{N})$, for which $\varrho_{D q \delta(\psi=1)} \geq \varrho_{D q \delta(\psi)} \forall(\psi \in \mathbb{N})$.

In the above explanation we defined q-RLDFWAA and q-RLDFOWAA operators. Now here we want to present q-rung linear Diophantine fuzzy hybrid weighted averaging aggregation (q-RLDFHWAA) operator.

Definition 12 Let $\varrho_{D q \psi}=\left\{\left(\left\langle{ }^{\psi} \wp_{D q},{ }^{\psi} \Re_{D q}\right\rangle,\left\langle{ }^{\psi} \alpha,{ }^{\psi} \beta\right\rangle\right)\right.$ : $\psi=1,2, \ldots n\}$ be an assembling of q-RLDFNs on the reference set $M$ and the weight vector $\Omega=\left(\Omega_{1}, \Omega_{2}, \ldots, \Omega_{n}\right)^{T}$ such that $\Omega>0$ with $\sum_{\psi=1}^{n} \Omega_{\psi}=1$, and $q \geq 1$; then the mapping $\theta: q-\operatorname{RLDFN}(M) \longrightarrow q-\operatorname{RLDFN}(M)$ is called q-rung linear Diophantine fuzzy hybrid weighted averaging aggregation (q-RLDFHWAA) operator and defined as;

$$
\begin{aligned}
q & -\operatorname{RLDFHWAA}\left(\varrho_{D q 1}, \varrho_{D q 2}, \varrho_{D q 3}, \ldots, \varrho_{D q n}\right) \\
& =\prod_{\psi=1}^{n}\left(\Omega_{\psi} \varrho_{(\delta) D q \psi}^{\diamond}\right. \\
& =\left(\begin{array}{c}
\left\langle\sqrt[q]{1-\prod_{\psi=1}^{n}\left(1-\psi \wp_{(\delta) D q}^{q \diamond}\right)^{\Omega_{\psi}}}, \prod_{\psi=1}^{n} \psi \Re_{(\delta) D q}^{\diamond \Omega_{\psi}}\right\rangle \\
\left\langle\sqrt[q]{1-\prod_{\psi=1}^{n}\left(1-\psi \alpha_{(\delta)}^{q \diamond}\right)^{\Omega_{\psi}}}, \prod_{\psi=1}^{n} \psi \beta_{(\delta)}^{\diamond \Omega_{\psi}}\right\rangle
\end{array}\right), q \geq 1 .
\end{aligned}
$$

Where $\rho_{\delta D q(\psi)}^{\diamond}$ is the $\psi$ th biggest weighted q-rung linear Diophantine fuzzy values $\varrho_{D q(i)}^{\diamond}\left(\varrho_{D q(\psi)}^{\diamond}=\left(\varrho_{D q(\psi)}\right)^{n \Omega_{\psi}}, \psi \in \mathbb{N}\right)$ and $\Omega=\left(\Omega_{1}, \Omega_{2}, \ldots, \Omega_{n}\right)^{T}$ be the weights of $\varrho_{D q(\psi)}^{\diamond}$ by mean of $\Omega>0$ with $\sum_{\psi=1}^{n} \Omega_{\psi}=1$.

If $\Omega=\left(\frac{1}{\Omega}, \frac{1}{\Omega}, \ldots, \frac{1}{\Omega}\right)$, then q-RLDFWAA and q-RLDFOWAA operators are considered to be a specific case q-RLDFHWAA. So it conclude that generalized form of q-RLDFWAA and q-RLDFOWAA operators is q-RLDFHWAA operator.

\section{5 q-Rung linear Diophantine fuzzy weighted geometric aggregation ( $q$-RLDFWGA) operator}

In this section, we present q-Rung linear Diophantine fuzzy weighted geometric aggregation (q-RLDFWGA), q-RLDFOWGA and q-RLDFHWGA operators. We also define certain score functions (SFs) and accuracy functions (AFs) for the comparative analysis in MADM of q-RLDFNs. Chen and Tan (1994) introduced the notion of score function for IFSs. Tversky and Kahneman (1992) have suggested a similar concept. The definition can be generalized to fuzzy numbers hybrid systems and q-RLDFNs. We more generalize the concept of score functions defined by $(1994 ; 1992)$, as; with the addition of qth power of reference parameters. Due to different strategies of different operators used in the algorithm, there are more than one mapping to find the value. To evaluate the actions of q-RLDFNs under the influence of these score functions, we describe different score functions in this manuscript and then compare their results.

Definition 13 Suppose $\varrho_{D q}=\left\{\left\langle\wp_{D q}, \Re_{D q}\right\rangle,\langle\alpha, \beta\rangle\right\}$ be a q-RLDFN, then score function (SF) on $\varrho_{D q}$ may be define by the transformation $\kappa: q-\operatorname{RLDFN}(M) \longrightarrow[-1,1]$ and given by

$\kappa_{\varrho_{D q}}=\kappa\left(\varrho_{D q}\right)=\left[\frac{\left(\wp_{D q}-\Re_{D q}\right)+\left(\alpha^{q}-\beta^{q}\right)}{2}\right] ; q \geq 1$

where $q-R L D F N(M)$ is an assembling of $q-R L D F N s$ on M.

Definition 14 The accuracy function $(\delta)$ may be defined by the transformation $\delta: q-R L D F N(M) \longrightarrow[0,1]$ and given as

$\delta_{\rho_{D q}}=\delta\left(\varrho_{D q}\right)=\left[\left(\frac{\wp_{D q}+\Re_{D q}}{4}\right)+\left(\frac{\alpha^{q}+\beta^{q}}{2}\right)\right] ; q \geq 1$

Definition 15 Let $\varrho_{D q 1}$ and $\varrho_{D q 2}$ be two $q-R L D F N s$ then by using the score and accuracy function we can easily compare these two $q$-RLDFNs as:

(i) if $\kappa_{\rho_{D q 1}}<\kappa_{\varrho_{D q 2}}$ then $\varrho_{D q 1}<\varrho_{D q 2}$,

(ii) if $\kappa_{\varrho_{D q 1}}>\kappa_{\varrho_{D q 2}}$ then $\varrho_{D q 1}>\varrho_{D q 2}$,

(iii) if $\kappa_{\varrho_{D q 1}}=\kappa_{\rho_{D q 2}}$ then we use accuracy function,

(a) if $\delta_{\rho_{D q 1}}<\delta_{\rho_{D q 2}}$ then $\rho_{D q 1}<\varrho_{D q 2}$,

(b) if $\delta_{\rho_{D q 1}}>\delta_{\rho_{D q 2}}$ then $\varrho_{D q 1}>\varrho_{D q 2}$,

(c) if $\delta_{\rho_{D q 1}}=\delta_{\rho_{D q 2}}$ then $\varrho_{D q 1} \approx \varrho_{D q 2}$.

Next definition is about quadratic score function

Definition 16 The quadratic score function (QSF) for $q-R L D F N$ is a mathematical transformation $\eta: q-\operatorname{RLDFN}(M) \longrightarrow[-1,1]$ and defined as

$\varpi_{\varrho_{D q}}=\varpi\left(\varrho_{D q}\right)=\left(\frac{\left(\wp_{D q}^{2}-\Re_{D q}^{2}\right)+\left(\left(\alpha^{q}\right)^{2}-\left(\beta^{q}\right)^{2}\right)}{2}\right) ; q \geq 1$

Definition 17 The quadratic accuracy function (QAF) for $q-R L D F N$ is a transformation $\eta: q-\operatorname{RLDFN}(M) \longrightarrow[0,1]$ and defined as 
$\chi_{\varrho_{D q}}=\chi\left(\varrho_{D q}\right)=\left[\left(\frac{\wp_{D q}^{2}+\Re_{D q}^{2}}{4}\right)+\left(\frac{\left(\alpha^{q}\right)^{2}+\left(\beta^{q}\right)^{2}}{2}\right)\right] ; q \geq 1$

Definition 18 Let $\varrho_{D q 1}$ and $\varrho_{D q 2}$ be two $q-R L D F N s$ then by using the quadratic score and accuracy function we can easily compare these two $q-R L D F N s$ as:

(i) if $\varpi_{\varrho_{D q 1}}<\varpi_{\rho_{D q 2}}$ then $\varrho_{D q 1}<\varrho_{D q 2}$,

(ii) if $\varpi_{\varrho_{D q 1}}>\varpi_{\varrho_{D q 2}}$ then $\varrho_{D q 1}>\varrho_{D q 2}$,

(iii) if $\varpi_{\varrho_{D q 1}}=\varpi_{\varrho_{D q 2}}$ then we use accuracy function,

(a) if $\chi_{\varrho_{D q 1}}<\chi_{\rho_{D q 2}}$ then $\varrho_{D q 1}<\varrho_{D q 2}$,

(b) if $\chi_{\rho_{D q 1}}>\chi_{\rho_{D q 2}}$ then $\varrho_{D q 1}>\rho_{D q 2}$,

(c) if $\chi_{\varrho_{D q 1}}=\chi_{\varrho_{D q 2}}$ then $\varrho_{D q 1} \approx \varrho_{D q 2}$.

Next we present another generalize score function known as expectation score function (ESF) .

Definition 19 Suppose $\varrho_{D q}=\left\{\left\langle\wp_{D q}, \Re_{D q}\right\rangle,\langle\alpha, \beta\rangle\right\}$ be a q-RLDFN, then expectation score function (ESF) on $\rho_{D q}$ can be define by the mapping $F: q-R L D F N(M) \longrightarrow[0,1]$ and given by

$$
\begin{aligned}
F_{\varrho_{D q}}= & F\left(\varrho_{D q}\right)=\left[\frac{\left(\wp_{D q}-\Re_{D q}+1\right)}{4}+\frac{\left(\alpha^{q}-\beta^{q}+1\right)}{4}\right] \\
& ; q \geq 1
\end{aligned}
$$

The ESF values are restricted by $[0,1]$ rather than $[-1,1]$. The ESF is generalized form of SF.

Now we are going to define q-RLDFWGA, q-RLDFOWGA and q-RLDFHWGA operators.

Definition 20 Let $\varrho_{D q \psi}=\left\{\left(\left\langle{ }^{\psi} \wp_{D q},{ }^{\psi} \Re_{D q}\right\rangle,\left\langle{ }^{\psi} \alpha,{ }^{\psi} \beta\right\rangle\right)\right.$ : $\psi=1,2, \ldots n\}$ be an assembling of q-RLDFNs on the reference set $M$ and the weight vector $\Omega=\left(\Omega_{1}, \Omega_{2}, \ldots, \Omega_{n}\right)^{T}$ with $\sum_{\psi=1}^{n} \Omega_{\psi}=1$ and $q \geq 1$, then transformation $\theta: q-R L D F N(M) \longrightarrow q-R L D F N(M)$ is called q-RLDFWGA operator and defined as

$$
\begin{aligned}
q- & R L D F W G A\left(\varrho_{D q 1}, \varrho_{D q 2}, \varrho_{D q 3}, \ldots, \varrho_{D q n}\right) \\
& =\prod_{\psi=1}^{n} \varrho_{D q \psi}^{\Omega_{\psi}} \\
& =\left(\begin{array}{c}
\left\langle\prod_{\psi=1}^{n} \psi \wp_{D q}^{\Omega_{\psi}}, \sqrt[q]{1-\prod_{\psi=1}^{n}\left(1-\psi \mathfrak{R}_{D q}^{q}\right)^{\Omega_{\psi}}}\right\rangle \\
\left\langle\prod_{\psi=1}^{n} \psi \alpha^{\Omega_{\psi}}, \sqrt[q]{1-\prod_{\psi=1}^{n}\left(1-\psi \beta^{q}\right)^{\Omega_{\psi}}}\right\rangle
\end{array}\right), q \geq 1 .
\end{aligned}
$$

This operator can easily be proof by using the q-RLDFS operations and by mathematical induction. In
q-RLDFWGA operator, $\varnothing$ denote positive membership and $\Re$ denote negative membership functions, $\alpha, \beta$ denote the reference parameters for both $\wp$ and $\Re$ respectively and $q \geq 1 . \Omega$ denote the weights, $\varrho_{D q \psi}$ are the q-RLDFNs, where $\psi \in \mathbb{N}$ and q-RLDFN( $M$ ) gathers all q-RLDFNs.

So, now let us we want to present q-RLDFOWGA operator.

Definition 21 Let $\varrho_{D q \psi}=\left\{\left(\left\langle\psi \wp_{D q},{ }^{\psi} \Re_{D q}\right\rangle,\left\langle{ }^{\psi} \alpha,{ }^{\psi} \beta\right\rangle\right)\right.$ : $\psi=1,2, \ldots n\}$ be an assembling of q-RLDFNs on the reference set $M$ and the weights $\Omega=\left(\Omega_{1}, \Omega_{2}, \ldots, \Omega_{n}\right)^{T}$ i.e. $\Omega>0$ with $\sum_{\psi=1}^{n} \Omega_{\psi}=1$ and $q \geq 1$, then the mapping $\theta: q-\operatorname{RLDFN}(M) \longrightarrow q-R \operatorname{LDFN}(M)$ is called q-rung linear Diophantine fuzzy ordered weighted geometric aggregation (q-RLDFOWGA) operator and defined as

$$
\begin{aligned}
q- & R L D F O W G A\left(\varrho_{D q 1}, \varrho_{D q 2}, \varrho_{D q 3}, \ldots, \varrho_{D q n}\right) \\
= & \prod_{\psi=1}^{n} \varrho_{D q \psi(\delta)}^{\Omega_{\psi}} \\
= & \left(\begin{array}{c}
\left\langle\prod_{\psi=1}^{n} \psi \wp_{D q(\delta)}^{\Omega_{\psi}}, \sqrt[q]{1-\prod_{\psi=1}^{n}\left(1-\psi \mathfrak{R}_{D q(\delta)}^{q}\right)^{\Omega_{\psi}}}\right\rangle \\
\left\langle\prod_{\psi=1}^{n} \psi \alpha_{(\delta)}^{\Omega_{\psi}}, \sqrt[q]{1-\prod_{\psi=1}^{n}\left(1-\psi \beta_{(\delta)}^{q}\right)^{\Omega_{\psi}}}\right\rangle
\end{array}\right), q \geq 1 .
\end{aligned}
$$

where $(\delta(1), \delta(2), \delta(3), \ldots, \delta(n))$ is the arrangement of $(\psi \in \mathbb{N})$, for which $\varrho_{D q \delta(\psi=1)} \geq \varrho_{D q \delta(\psi)} \forall(\psi \in \mathbb{N})$.

In the above explanation we defined q-RLDFWGA and q-RLDFOWGA operators. Now here we want to present q-RLDFHWGA operator.

Definition 22 Let $\varrho_{D q \psi}=\left\{\left(\left\langle{ }^{\psi} \wp_{D q},{ }^{\psi} \mathfrak{R}_{D q}\right\rangle,\left\langle{ }^{\psi} \alpha,{ }^{\psi} \beta\right\rangle\right)\right.$ : $\psi=1,2, \ldots n\}$ be an assembling of q-RLDFNs on the reference set $M$ and the weights $\Omega=\left(\Omega_{1}, \Omega_{2}, \ldots, \Omega_{n}\right)^{T}$ i.e. $\Omega>0$ with $\sum_{\psi=1}^{n} \Omega_{\psi}=1$ and $q \geq 1$, then $\theta: q-\operatorname{RLDFN}(M) \longrightarrow$ $q-\operatorname{RLDFN}(M)$ is called q-rung linear Diophantine fuzzy hybrid weighted geometric aggregation (q-RLDFHWGA) operator and defined as;

$$
\begin{aligned}
q- & R L D F H W G A\left(\varrho_{D q 1}, \varrho_{D q 2}, \varrho_{D q 3}, \ldots, \varrho_{D q n}\right) \\
= & \prod_{\psi=1}^{n} \varrho_{(\delta) D q \psi}^{\diamond \Omega_{\psi}} \\
= & \left(\begin{array}{c}
\left\langle\prod_{\psi=1}^{n} \psi \wp_{(\delta) D q}^{\diamond \Omega_{\psi}}, \sqrt[q]{1-\prod_{\psi=1}^{n}\left(1-\psi \mathfrak{R}_{(\delta) D q}^{q \diamond}\right)^{\Omega_{\psi}}}\right\rangle \\
\left\langle\prod_{\psi=1}^{n} \psi \alpha_{(\delta)}^{\diamond \Omega_{\psi}}, \sqrt[q]{1-\prod_{\psi=1}^{n}\left(1-\psi \beta_{(\delta)}^{q \diamond}\right)^{\Omega_{\psi}}}\right\rangle
\end{array}\right), q \geq 1 .
\end{aligned}
$$


Where $\varrho_{\delta D q(\psi)}^{\diamond}$ is the $\psi$ th biggest weighted q-RLDF values $\rho_{D q(i)}^{\diamond}\left(\rho_{D q(\psi)}^{\diamond}=\left(\varrho_{D q(\psi)}\right)^{n \Omega_{\psi}}, \psi \in \mathbb{N}\right)$ and $\Omega=\left(\Omega_{1}, \Omega_{2}, \ldots, \Omega_{n}\right)^{T}$ be the weights of $\varrho_{D q(\psi)}^{\diamond}$ by mean of $\Omega>0$ with $\sum_{\psi=1}^{n} \Omega_{\psi}=1$

If $\Omega=\left(\frac{1}{\Omega}, \frac{1}{\Omega}, \ldots, \frac{1}{\Omega}\right)$, then q-RLDFWGA and q-RLDFOWGA operators are considered to be a specific case q-RLDFHWGA. So it conclude that generalized form of q-RLDFWGA and q-RLDFOWGA operators is q-RLDFHWGA operator.

\section{Model for MADM using q-RLDF data}

We introduce a new approach to MADM throughout this portion under the q-RLDFS environment which is q-RLDFWA, q-RLDFOWA and q-RLDFHWA operators and q-RLDFWGA, q-RLDFOWGA and q-RLDFHWGA operators. So for this we present algorithms for same numerical model and by using of various types of SFs we get preventive and control rankings for final decision about COVID-19 outbreak.

\section{Case study}

Within this section, a specific case is presented on emergency public health decision-making to convey the implementation of the proposed model. We are suggesting a novel COVID-19 outbreak solution (Chan et al. 2020; Wu and McGoogan 2020; WHO 2020a; Yu et al. 2020; Baharoon and Memish 2019; WHO 2020b, c) to determine the best attribute under q-RLDF information. The frequent outbreak of emergency incidents in last few years has caused enormous losses for the human community. Whenever the incidents happened, quick monitoring and implementation of one of the emergency alternatives is necessary. Based on the above study, a framework of q-RLDF emergency decision-making is applied to explain the process of emergency decision-making for COVID-19. And to study the prevention and control of Corona virus and also to overcome the spreading of this disease.

Problem Description: The novel coronavirus forced the Chinese government to launch the largest quarantine order in human history at the beginning of 2020, affecting approximately 45 million people. WHO has tentatively nominated the virus as the novel coron-avirus of 2019 (2019-nCoV). In December 2019, the 2019 coronavirus disease caused by the COVID-19 virus was first identified in wuhan, China. On 30 January 2020, the DirectorGeneral of the WHO (World Health Organization) reported that the current outbreak constituted a global public health emergency. As of April 8th, 2020, more than 1,518,614
(COVID-19 Global cases (JHU) 2020) cases have been confirmed in 209 countries and territories, of which 48,079 (COVID-19 Global cases (JHU) 2020) were classified as serious. At least 88,480 (COVID-19 Global cases (JHU) 2020) deaths have been attributed to the disease, most in mainland china, italy, iran, USA, UK e.t.c with more than 1000 deaths in other countries. More than 330,357 (COVID-19 Global cases (JHU) 2020) people have recovered. The risk of it spreading further is very high. No doubt this disease has caused enormous economic losses, environmental pollution, insufficient of PPE (personal protective equipment); PPE includes gloves, medical masks, goggles or a face shield. In addition, the capacity to expand PPE production is insufficient, and the global request for respirators and masks can not be achieved, especially if the widespread, improper use of PPE continues.

WHO is working with international expert networks and laboratory partnerships, infection prevention and control, clinical management and mathematical modelling.

In these situations, finding an effective way of emergency response is necessary in order to prevent further casualties and save people's lives. For both healthcare and community settings, preventive and mitigative measures are necessary. Here is how we are using our new q-RLDFS framework for the DM. Based on the q-RLDFWA and q-RLDFWGA operators we propose a procedure for a decision maker to select the best choice with q-RLDF information.

Table 6 show the various alternatives for Corona virus prevention and control.

There are five basic Public health emergency factor to reduce the general risk of this disease. The most effective preventive measure in the community include the following:

(1) $\breve{G}_{1}$ : Clinical management: Once the virus spread, Vaccination is an extremely effective way to avoid certain infectious diseases. Vaccines are generally very safe, and serious adverse reactions are uncommon. No specific treatment of COVID-19 is currently available. Clinical management includes prompt implementation of recommended infection prevention and control measure and supportive management of complication, including advanced organ support if indicated.

(2) $\breve{G}_{2}$ : First-Aid training or increased Personal Protective equipment: This disease spread very quickly, so to control on this viral virus first, trained or Avoid people of this disease symptoms. So it is strongly advised that people attend a fully regulated practical or online first Aid course to understand what to do in a medical emergency. Another issue is the shortage of test kits. The situation will be improved with increased production of test kits, lack of confirmation requirements and local government decision to threaten and finally quarantine of all suspected cases. Sending masks, gloves, respirators and gowns to countries in every region. Face masks provide limited protection in preventing some 
Table 6 Prevention alternatives of viral (COVID-19) disease (infectious)

\begin{tabular}{|c|c|c|}
\hline Notation & Alternatives & Description \\
\hline$M_{1}$ & Online first-Aid course & $\begin{array}{l}\text { Creating online first-aid courses which will } \\
\text { aware people to know about their duties and } \\
\text { responsibilities during COVID-19 } \\
\text { Keep updated about COVID-19 via official } \\
\text { website such as advisories from UNICEF, the } \\
\text { WHO and the national health ministry }\end{array}$ \\
\hline$M_{2}$ & Vaccination & $\begin{array}{l}\text { Actually COVID-19 vaccine is not available } \\
\text { There are currently several clinical trials } \\
\text { being performed to test possible COVID-19 } \\
\text { therapies }\end{array}$ \\
\hline$M_{3}$ & Local governments decision & $\begin{array}{l}\text { For safety of local people, the decision of local } \\
\text { government would be essential and helpful. } \\
\text { Stay at home, stay away from others, contact } \\
\text { the doctor if you develop symptoms } \\
\text { To prevent the transmission of the infection } \\
\text { isolate the affected or suspected person from } \\
\text { other healthy people } \\
\text { Follow government advise to prevent spread of } \\
\text { COVID-19. People may follow such steps as } \\
\text { per health care safety }\end{array}$ \\
\hline$M_{4}$ & Medical support & $\begin{array}{l}\text { Establishment of medical support is mandatory } \\
\text { Medical support can provide mask, gloves, } \\
\text { face mask and gowns to reduce the spread of } \\
\text { the disease to all members of society } \\
\text { Increasing COVID-19 awareness } \\
\text { Wash hand often with soap and water for at } \\
\text { least } 20 \text { sec } \\
\text { Used an alcohol based hand sanitizer with at } \\
\text { least } 60 \% \text { alcohol } \\
\text { Avoid touching the eyes, nose and mouth } \\
\text { Make personal and professional adjustments as } \\
\text { more is known about the COVID-19 situation } \\
\text { Do not share cups, eating intensely, food or } \\
\text { drinks with others }\end{array}$ \\
\hline
\end{tabular}

one infected from spreading the virus. Therefore, the easiest way to prevent spread, is by good personal hygiene. However, the world is facing severe disruption in the marked for PPE.

(3) $\breve{G}_{3}$ : Trained Technician: It is extremely quick to share the genetic makeup of the virus to enable the rest of the world begin developing specific screening and start working on potential vaccines.

(4) $\breve{G}_{4}$ : Banned intra-City Transportation: The disease caused by the virus is serious. For safety of local people it is necessary for local government that take step or announced that intra-city travel has been banned to compel patients to go to nearby community clinics. And also suspended all flights and train services from and to wuhan and cancelled their lunar new year celebrations, and also need to maintain at least 1 meter (3 feet) distance between yourself and anyone who is coughing or sneezing.

(5) $\breve{G}_{5}$ : Global uncertainty; The economic fallout from coronavirus: The sharp downturn in the transportation and hospitality sectors will affect the overall economy and during the first quarter, consumption and trade will also affect. It may significantly affect the country,s overall economic situation. It has implications, not just for china, but for the entire world. The world depend on Chinese growth. The novel coronavirus has badly affected on global demand for oil. Factory shutdown delays goods and parts supply from China, impacting companies worldwide including Apple and Nissan.

Figure 4 presented the flow chart of the proposed method. We now build the input information in the q-RLDFS framework, where positive membership and negative membership functions $\left\langle\wp_{D q}, \mathfrak{R}_{D q}\right\rangle$ tells us about the satisfaction and dissatisfaction of optimal attributes and reference parameters $\langle\alpha, \beta\rangle$ represent the "the most important public health emergency response" and "less important public health emergency response". Now we are able to collect some data on the basis of q-RLDFSs about the important factors in evaluating emergency response requirements for public health. If 
we select three health experts, who control the entire emergency response process for public health then we have three q-RLDFSs for the input data, given in Tables 6, 7 and 8.

Suppose there are four emergency alternatives, each represented by $M_{i}(i=1,2,3,4)$ respectively. Three health experts, $D_{k}(k=1,2,3)$ who control the entire emergency response process for public health, the emergency alternatives are appointed to be evaluated under five factors/criteria of $\breve{G}_{j}(j=1,2,3,4,5)$ as explained above. In order to choose the effective alternative, the proposed method addresses the problem of emergency decision-making. The following steps shall be required to evaluate the four emergency alternatives $M_{i}=(i=1,2,3,4)$.

\subsection{Mathematical modeling}

This subsection is about construction of algorithms. Throughout this field we develop algorithms depending on the q-RLDFWAA and the q-RLDFWGA operators. Using various score functions, we calculate scores and finally make a comparison the values obtained from these techniques. The purpose for presenting these two different algorithms is that the theory of q-RLDFS and its flexibility to be used in various circumstances.

Here the MADM problem was used to reduce the general risk of this disease with q-RLDF data in the community. Consider $M=\left\{M_{1}, M_{2}, M_{3}, \ldots, M_{m}\right\}$ be a set of
Fig. 4 Flow chart of the proposed method

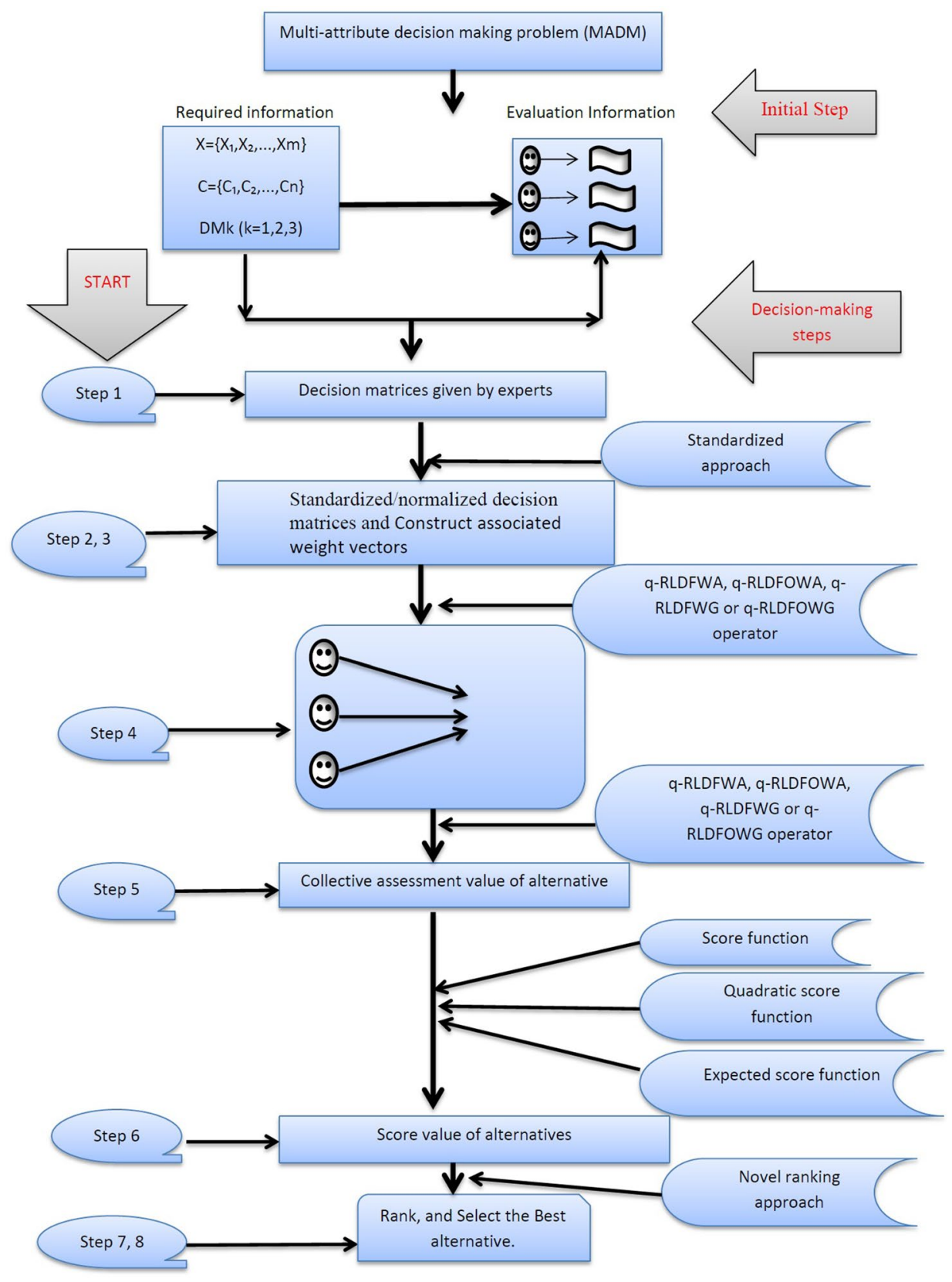


alternatives and $\breve{G}=\left\{\breve{G}_{1}, \breve{G}_{2}, \breve{G}_{3}, \ldots \breve{G}_{n}\right\}$ a set of criteria. Let $\Omega=\left(\Omega_{1}, \Omega_{2}, \ldots, \Omega_{n}\right)^{T}$ be weight of attribute/criteria $\Omega_{\psi}(\psi=1,2, \ldots, n)$ such that $\Omega_{\psi}>0$ with $\sum_{\psi=1}^{n} \Omega_{\psi}=1$. Each decision maker provides his own payoff matrix table, the results are q-RLDF numbers. Consider that $D M=\left(\left\langle{ }^{g \psi} \wp_{D q K},{ }^{g \psi} \mathfrak{R}_{D q K}\right\rangle,\left\langle{ }^{g \psi} \alpha,{ }^{g \psi} \beta\right\rangle\right)_{m \times n}$ are the q-RLDF decision matrix, where ${ }^{g \psi} \wp_{D q K}$ is the positive membership function, ${ }^{g \psi} \mathfrak{R}_{D q K}$ is the negative membership function and ${ }^{g \psi} \alpha,{ }^{g \psi} \beta$ are the references parameters for which the alternative $\left(M_{\psi}\right)$ satisfies the $\left(\breve{G}_{\psi}\right)$ attribute provided by the decision-makers, where ${ }^{g \psi} \wp_{D q},{ }^{g \psi}{ }^{q} \mathfrak{R}_{D q},{ }^{g \psi} \alpha,{ }^{g \psi} \beta \subset[0,1]$ such that $0 \leq{ }^{g \psi}\left((\alpha)^{q} \wp_{D q}\right)+{ }^{g \psi}\left((\beta)^{q} \boldsymbol{R}_{D q}\right) \leq 1,(g=1,2, \ldots, m)$.On the ground of above analysis we do your best to solve following new algorithm and process of MADM problem with q-RLDFNs by mean of q-RLDFWA, q-RLDFOWA, q-RLDFHWA, q-RLDFWG, q-RLDFOWG and q-RLDFHWG.

Algorithm-1 Algorithm based on q-RLDFWA Aggregation operator

\section{Input:}

Step 1: Establish a DMs team of q-RLDF-data in q-RLDFSs format for an appropriate number of alternatives and attributes $T_{D q \psi} ;(\psi \in \mathbb{N})$. Here $D M=\left\{D M_{1}, D M_{2}, \ldots, D M_{u}, D M_{l}\right\}$ denotes a decision maker (DMrs) group. The preferences of each DM are calculated via q-RLDFNs. So employ the decision data defined in matrix DM, which are q-RLDFNs and are shown in the shape of decision matrix $(D M) D M_{1}, D M_{2}$ and $\mathrm{DM}_{3}$ with weighting vector $\Omega$.

Step 2: Standardized q-RLDF-data input:

\begin{tabular}{|c|c|c|c|}
\hline$q=3$ & $\breve{G}_{1}$ & $\breve{G}_{2}$ & $\breve{G}_{3}$ \\
\hline$M_{1}$ & $\left(\begin{array}{l}\langle .8, .9\rangle, \\
\langle .85, .7\rangle\end{array}\right)$ & $\left(\begin{array}{c}\langle .9, .88\rangle, \\
\langle .8, .7\rangle\end{array}\right)$ & $\left(\begin{array}{l}\langle 1,1\rangle, \\
\langle .7, .8\rangle\end{array}\right)$ \\
\hline$M_{2}$ & $\left(\begin{array}{l}\langle 1,1\rangle, \\
\langle .7, .8\rangle\end{array}\right)$ & $\left(\begin{array}{c}\langle .98, .89\rangle, \\
\langle .8, .7\rangle\end{array}\right)$ & $\left(\begin{array}{c}\langle .87, .95\rangle, \\
\langle .8, .7\rangle\end{array}\right)$ \\
\hline$M_{3}$ & $\left(\begin{array}{l}\langle .8, .7\rangle, \\
\langle .6, .9\rangle\end{array}\right)$ & $\left(\begin{array}{l}\langle 1, .8\rangle, \\
\langle .7, .8\rangle\end{array}\right)$ & $\left(\begin{array}{c}\langle .94, .84\rangle, \\
\langle .8, .7\rangle\end{array}\right)$ \\
\hline$M_{4}$ & $\left(\begin{array}{l}\langle .9,1\rangle, \\
\langle .9, .6\rangle\end{array}\right)$ & $\left(\begin{array}{l}\langle 1,0.9\rangle, \\
\langle .7, .85\rangle\end{array}\right)$ & $\left(\begin{array}{c}\langle .95, .88\rangle, \\
\langle .8, .7\rangle\end{array}\right)$ \\
\hline$M_{1}$ & $\begin{array}{l}\breve{G}_{4} \\
\left(\begin{array}{l}\langle .9,1\rangle, \\
\langle .9, .6\rangle\end{array}\right)\end{array}$ & $\begin{array}{l}\breve{G}_{5} \\
\left(\begin{array}{l}\langle .8, .9\rangle, \\
\langle .7, .85\rangle\end{array}\right)\end{array}$ & \\
\hline$M_{2}$ & $\left(\begin{array}{c}\langle .82,1\rangle, \\
\langle .8, .7\rangle\end{array}\right)$ & $\left(\begin{array}{l}\langle 1, .9\rangle, \\
\langle .6, .9\rangle\end{array}\right)$ & \\
\hline$M_{3}$ & $\left(\begin{array}{l}\langle .9,1\rangle, \\
\langle .8, .7\rangle\end{array}\right)$ & $\left(\begin{array}{c}\langle .88, .96\rangle, \\
\langle .8, .7\rangle\end{array}\right)$ & \\
\hline$M_{4}$ & $\left(\begin{array}{l}\langle .9, .8\rangle, \\
\langle .7, .85\rangle\end{array}\right)$ & $\left(\begin{array}{c}\langle .9,1\rangle, \\
\langle .85, .7\rangle\end{array}\right)$ & \\
\hline
\end{tabular}

\begin{tabular}{|c|c|c|c|}
\hline$q=3$ & $\breve{G}_{1}$ & $\breve{G}_{2}$ & $\breve{G}_{3}$ \\
\hline$M_{1}$ & $\left(\begin{array}{l}\langle 1,1\rangle, \\
\langle .7,8\rangle\end{array}\right)$ & $\left(\begin{array}{l}\langle .9,1\rangle, \\
\langle .8, .7\rangle\end{array}\right)$ & $\left(\begin{array}{c}\langle .95, .85\rangle, \\
\langle .7, .8\rangle\end{array}\right)$ \\
\hline$M_{2}$ & $\left(\begin{array}{c}\langle .99, .88\rangle, \\
\langle .7, .85\rangle\end{array}\right)$ & $\left(\begin{array}{c}\langle 1,1\rangle, \\
\langle .8, .7\rangle\end{array}\right)$ & $\left(\begin{array}{l}\langle .8,1\rangle, \\
\langle .8, .7\rangle\end{array}\right)$ \\
\hline$M_{3}$ & $\left(\begin{array}{c}\langle .85, .88\rangle, \\
\langle .6, .9\rangle\end{array}\right)$ & $\left(\begin{array}{c}\langle .8, .9\rangle, \\
\langle .85, .7\rangle\end{array}\right)$ & $\left(\begin{array}{c}\langle .95, .85\rangle \\
\langle .7, .8\rangle\end{array}\right)$ \\
\hline$M_{4}$ & $\left(\begin{array}{l}\langle 1,1\rangle, \\
\langle .7,8\rangle\end{array}\right)$ & $\left(\begin{array}{l}\langle .9,1\rangle, \\
\langle .8, .7\rangle\end{array}\right)$ & $\left(\begin{array}{c}\langle .95, .9\rangle, \\
\langle .8, .7\rangle\end{array}\right)$ \\
\hline & $\breve{G}_{4}$ & $\breve{G}_{5}$ & \\
\hline$M_{1}$ & $\left(\begin{array}{c}\langle .88, .95\rangle, \\
\langle .8, .7\rangle\end{array}\right)$ & $\left(\begin{array}{c}\langle .8, .95\rangle, \\
\langle .7, .85\rangle\end{array}\right)$ & \\
\hline$M_{2}$ & $\left(\begin{array}{l}\langle 1, .9\rangle, \\
\langle .7, .8\rangle\end{array}\right)$ & $\left(\begin{array}{l}\langle 1, .9\rangle, \\
\langle .6, .9\rangle\end{array}\right)$ & \\
\hline$M_{3}$ & $\left(\begin{array}{l}\langle .9,1\rangle, \\
\langle .8, .7\rangle\end{array}\right)$ & $\left(\begin{array}{c}\langle .88, .96\rangle, \\
\langle .8, .7\rangle\end{array}\right)$ & \\
\hline$M_{4}$ & $\left(\begin{array}{c}\langle .87, .95\rangle, \\
\langle .8, .7\rangle\end{array}\right)$ & $\left(\begin{array}{l}\langle .9,1\rangle, \\
\langle .9, .6\rangle\end{array}\right)$ & \\
\hline
\end{tabular}

Before further calculations, it is important to normalize the input data in order to achieve the best and most accurate solutions. As a result, the q-RLDF analysis can be standardized by

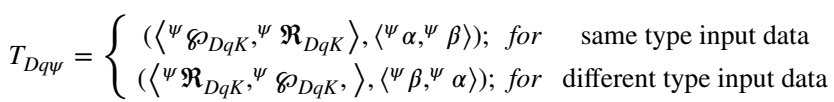

In this case, the input data for all attributes are the same, then we don't require to normalize the data. All alternatives and criteria in our given problem are of the same types.

Step 3: We obtain for each decision-maker $D$ the equivalent weights ${ }^{\zeta} \ell$ to give priority to each decision-maker's opinion, according to the $\zeta$ decision-makers; $\zeta=1,2, \ldots, q$.

Calculations:

Step 4: Evaluate the final weight

$\ell=\left(\frac{1}{q} \sum_{\zeta=1}^{q}{ }^{\zeta} \ell_{1}, \frac{1}{q} \sum_{\zeta=1}^{q}{ }^{\zeta} \ell_{2}, \ldots, \frac{1}{q} \sum_{\zeta=1}^{q}{ }^{\zeta} \ell_{n}\right)^{T}$

Step 5: Utilize the decision information given in matrix $D M_{k}(k=1,2,3)$ into the collective (combine) q-RLDF $D M$ by applying the q-RLDF operators with associated weights $\Omega_{\psi}(\psi=1,2,3)$ of attribute $\breve{G}_{j}$ 


$$
\begin{aligned}
& q-R L D F W A A\left(\varrho_{D q 1}, \varrho_{D q 2}, \varrho_{D q 3}, \ldots, \varrho_{D q n}\right) \\
& =\prod_{\psi=1}^{n}\left(\Omega_{g \psi} \rho_{D q g \psi}\right) \\
& =\left(\begin{array}{c}
\left\langle\sqrt[q]{1-\prod_{\psi=1}^{n}\left(1-\psi \wp_{D q}^{q}\right)^{\Omega_{\psi}}}, \prod_{\psi=1}^{n} \psi \boldsymbol{R}_{D q}^{\Omega_{\psi}}\right\rangle \\
\left\langle\sqrt[q]{1-\prod_{\psi=1}^{n}\left(1-\psi \alpha^{q}\right)^{\Omega_{\psi}}, \prod_{\psi=1}^{n} \psi \beta^{\Omega_{\psi}}}\right\rangle
\end{array}\right) \\
& q-R L D F O W A A\left(\varrho_{D q 1}, \varrho_{D q 2}, \varrho_{D q 3}, \ldots, \varrho_{D q n}\right) \\
& =\prod_{\psi=1}^{n}\left(\Omega_{g \psi} \varrho_{D q g \psi(\delta)}\right) \\
& =\left(\begin{array}{c}
\left\langle\sqrt[q]{\left.1-\prod_{\psi=1}^{n}\left(1-\psi \wp_{D q(\delta)}^{q}\right)^{\Omega_{\psi}}, \prod_{\psi=1}^{n}{ }^{\psi} \Re_{D q(\delta)}^{\Omega_{\psi}}\right\rangle}\right\rangle, \\
\left\langle\sqrt[q]{\left.1-\prod_{\psi=1}^{n}\left(1-\psi \alpha_{(\delta)}^{q}\right)^{\Omega_{\psi}}, \prod_{\psi=1}^{n}{ }^{\psi} \beta_{(\delta)}^{\Omega_{\psi}},\right\rangle}\right)
\end{array}\right) \\
& q-R L D F H W A A\left(\varrho_{D q 1}, \varrho_{D q 2}, \varrho_{D q 3}, \ldots, \varrho_{D q n}\right) \\
& =\prod_{\psi=1}^{n}\left(\Omega_{g \psi} \varrho_{(\delta) D q g \psi}^{\diamond}\right. \\
& =\left(\begin{array}{c}
\left\langle\sqrt[q]{1-\prod_{\psi=1}^{n}\left(1-\psi \wp_{(\delta) D q}^{q \diamond}\right)^{\Omega_{\psi}}}, \prod_{\psi=1}^{n} \psi \mathfrak{R}_{(\delta) D q}^{\diamond \Omega_{\psi}}\right\rangle, \\
\left\langle\sqrt[q]{1-\prod_{\psi=1}^{n}\left(1-\psi \alpha_{(\delta)}^{q \diamond}\right)^{\Omega_{\psi}}, \prod_{\psi=1}^{n} \psi} \beta_{(\delta)}^{\diamond \Omega_{\psi}}\right\rangle
\end{array}\right)
\end{aligned}
$$$$
q-R L D F W G A\left(\varrho_{D q 1}, \varrho_{D q 2}, \varrho_{D q 3}, \ldots, \varrho_{D q n}\right)
$$$$
=\prod_{\psi=1}^{n} \varrho_{D q g \psi}^{\Omega_{g \psi}}
$$$$
=\left(\begin{array}{c}
\left\langle\prod_{\psi=1}^{n} \psi \wp_{D q}^{\Omega_{\psi}}, \sqrt[q]{1-\prod_{\psi=1}^{n}\left(1-\psi \mathfrak{R}_{D q}^{q}\right)^{\Omega_{\psi}}}\right\rangle, \\
\left\langle\prod_{\psi=1}^{n} \psi \alpha^{\Omega_{\psi}}, \sqrt[q]{1-\prod_{\psi=1}^{n}\left(1-\psi \beta^{q}\right)^{\Omega_{\psi}}}\right\rangle
\end{array}\right)
$$$$
q-\operatorname{RLDFOWGA}\left(\varrho_{D q 1}, \varrho_{D q 2}, \varrho_{D q 3}, \ldots, \varrho_{D q n}\right)
$$$$
=\prod_{\psi=1}^{n} \varrho_{D q g \psi(\delta)}^{\Omega_{g \psi}}
$$$$
=\left(\begin{array}{c}
\left\langle\prod_{\psi=1}^{n}{ }^{\psi} \wp_{D q(\delta)}^{\Omega_{\psi}}, \sqrt[q]{1-\prod_{\psi=1}^{n}\left(1-\psi \mathfrak{R}_{D q(\delta)}^{q}\right)^{\Omega_{\psi}}}\right\rangle \\
\left\langle\prod_{\psi=1}^{n} \psi \alpha_{(\delta)}^{\Omega_{\psi}}, \sqrt[q]{1-\prod_{\psi=1}^{n}\left(1-\psi \beta_{(\delta)}^{q}\right)^{\Omega_{\psi}}}\right\rangle
\end{array}\right)
$$

$$
\begin{aligned}
q- & \operatorname{RLDFHWGA}\left(\varrho_{D q 1}, \varrho_{D q 2}, \varrho_{D q 3}, \ldots, \varrho_{D q n}\right) \\
& =\prod_{\psi=1}^{n} \varrho_{(\delta) D q g \psi}^{\diamond \Omega_{g \psi}} \\
& =\left(\begin{array}{c}
\left\langle\prod_{\psi=1}^{n} \psi \wp_{(\delta) D q}^{\diamond \Omega_{\psi}}, \sqrt[q]{1-\prod_{\psi=1}^{n}\left(1-\psi \mathfrak{R}_{(\delta) D q}^{q \diamond}\right)^{\Omega_{\psi}}}\right\rangle, \\
\left\langle\prod_{\psi=1}^{n} \psi \alpha_{(\delta)}^{\diamond \Omega_{\psi}}, \sqrt[q]{1-\prod_{\psi=1}^{n}\left(1-\psi \beta_{(\delta)}^{q \diamond}\right)^{\Omega_{\psi}}}\right\rangle
\end{array}\right)
\end{aligned}
$$

to obtained the overall preference values of the alternatives $M_{g}$.

Step 6: Again compute collective aggregated value for each attributes $\sigma(\hbar)$ with weight vector $\Omega_{\psi}(\psi=1,2,3,4,5)$ by using eq 7.1, 7.2, 7.3, 7.4, 7.5 and 7.6 of q-RLDF operators from above definition

Step 7: From aggregated values calculate scores of each attributes $\sigma(\hbar)$ using score, quadratic, and expectation score functions by using above definitions of graded function.

\section{Output:}

Step 8: Rank the attribute on the basis of score, quadratic, and expectation score functions values separately.

Step 9: Higher-score attribute has the maximum rank and must be selected for final decision.

End.

\section{Solution by using Algorithm 1:}

Step-1: We're applying our input data over this algorithm. Three health experts are appointed to evaluate the four alternatives for emergencies $M_{i}(i=1,2,3,4)$ with respect to five attributes $\breve{G}_{j}(j=1,2,3,4,5)$, and the decision matrices $D_{k}(k=1,2,3)$ are constructed as shown in Tables 7, 8 and 9 .

Step-2: We complete the data first by building the weights that correspond to each decision-maker. This stage indicates that every health expert's opinion is valuable for final decision. The three weights for three q-RLDF data are given as

Health Expert 1 opinion ${ }^{1} \ell=(0.3,0.45,0.25)^{T}$

Health Expert 2 opinion ${ }^{2} \ell=(0.5,0.3,0.2)^{T}$

Health Expert 3 opinion ${ }^{3} \ell=(0.4,0.3,0.3)^{T}$

Using step $4::$ in above Algorithm, we find the final set of weights, so we get $\ell=(0.4,0.35,0.25)^{T}$. It is clear from final weight vector that $\sum_{\zeta=1}^{3} \ell \zeta=1$.

Step-3: Now we use q-RLDFWA, q-RLDFOWA, and q-RLDFHWA operators to calculate the collective (combine) q-RLDF information, given in Table 10. 
Step-4: Again, if q-RLDFOWA is used, then same process can be apply as we did in q-RLDFWA but in current method we first find order of each attributes of three experts individually by using SF, QSF, ESF. The fundamental aspect of the OWA operator is the reordering step; it first reorders all the given input data (attributes) in descending order and then weights these ordered input data (attributes), and finally

\begin{tabular}{|c|c|c|c|}
\hline$q=3$ & $\breve{G}_{1}$ & $\breve{G}_{2}$ & $\breve{G}_{3}$ \\
\hline$M_{1}$ & $\left(\begin{array}{l}\langle .9,1\rangle, \\
\langle .8, .7\rangle\end{array}\right)$ & $\left(\begin{array}{c}\langle .88, .9\rangle, \\
\langle .6, .9\rangle\end{array}\right)$ & $\left(\begin{array}{c}\langle .8, .9\rangle, \\
\langle .7, .85\rangle\end{array}\right)$ \\
\hline$M_{2}$ & $\left(\begin{array}{l}\langle 1, .9\rangle, \\
\langle .7, .8\rangle\end{array}\right)$ & $\left(\begin{array}{c}\langle .95, .78\rangle, \\
\langle .8, .78\rangle\end{array}\right)$ & $\left(\begin{array}{c}\langle .85,1\rangle, \\
\langle .7, .8\rangle\end{array}\right)$ \\
\hline$M_{3}$ & $\left(\begin{array}{l}\langle .8,1\rangle, \\
\langle .8, .7\rangle\end{array}\right)$ & $\left(\begin{array}{l}\langle 1,1\rangle, \\
\langle .7, .8\rangle\end{array}\right)$ & $\left(\begin{array}{c}\langle .8, .9\rangle, \\
\langle .7, .85\rangle\end{array}\right)$ \\
\hline$M_{4}$ & $\left(\begin{array}{c}\langle .9, .87\rangle, \\
\langle .6, .9\rangle\end{array}\right)$ & $\left(\begin{array}{l}\langle 1, .9\rangle, \\
\langle .6, .9\rangle\end{array}\right)$ & $\left(\begin{array}{l}\langle 1,1\rangle, \\
\langle .8, .7\rangle\end{array}\right)$ \\
\hline & $\breve{G}_{4}$ & $\breve{G}_{5}$ & \\
\hline$M_{1}$ & $\left(\begin{array}{c}\langle .85, .87\rangle, \\
\langle .7, .85\rangle\end{array}\right)$ & $\left(\begin{array}{c}\langle .95, .85\rangle, \\
\langle .7, .8\rangle\end{array}\right)$ & \\
\hline$M_{2}$ & $\left(\begin{array}{c}\langle 1, .8\rangle, \\
\langle .7, .8\rangle\end{array}\right)$ & $\left(\begin{array}{l}\langle 1, .9\rangle, \\
\langle .6, .9\rangle\end{array}\right)$ & \\
\hline$M_{3}$ & $\left(\begin{array}{c}\langle .87, .95\rangle, \\
\langle .8, .7\rangle\end{array}\right)$ & $\left(\begin{array}{c}\langle .88, .96\rangle, \\
\langle .8, .7\rangle\end{array}\right)$ & \\
\hline$M_{4}$ & $\left(\begin{array}{c}\langle .98, .89\rangle, \\
\langle .7, .8\rangle\end{array}\right)$ & $\left(\begin{array}{l}\langle 1, .9\rangle, \\
\langle .6, .9\rangle\end{array}\right)$ & \\
\hline
\end{tabular}

aggregates all these ordered weighted attributes into a collective one. We repeat same steps of above Algorithm.

Step-5: Again, we construct the weight vectors by using step 4 of this algorithm, corresponding to the collective q-RLDFWA decision matrix. Let the five weight vectors for Table 10 of q-RLDF data are given as

$$
\begin{aligned}
& { }^{1} \ell=(0.3,0.25,0.2,0.15,0.1)^{T} \\
& { }^{2} \ell=(0.25,0.35,0.2,0.1,0.1)^{T} \\
& { }^{3} \ell=(0.35,0.25,0.15,0.15,0.1)^{T} \\
& { }^{4} \ell=(0.3,0.3,0.2,0.1,0.1)^{T} \\
& { }^{5} \ell=(0.4,0.2,0.1,0.2,0.1)^{T}
\end{aligned}
$$

We calculate these weights by using step 4, we obtained the final weight vectors given as $\ell=(0.32,0.27,0.17,0.14,0.1)^{T}$. It is clear from final weight vectors that $\sum_{\zeta=1}^{5} \ell \zeta=1$.

Step-6: We again repeat above step and aggregate Table 10 alternative wise by using eq 7.1, 7.2 and 7.3 with weights $\ell=(0.32,0.27,0.17,0.14,0.1)^{T}$, So we get q-RLDFWA, q-RLDFOWA, q-RLDFHWA $D M$ as described in Tables 11, 12 and 13.

Step-7: After that, we determine the value of its score by using SF, QSF and ESF via Definition (16-22). we get Table 14;

Step-8: Next we ranked (Table 14) the attributes of q-RLDFWA, q-RLDFOWA and q-RLDFHWA, we get the final result shown in Table 15;

Step-9: From this we conclude that; $M_{4}$ is chose the best attribute (method) for Corona virus prevention and control.
Table 10 The collective q-RLDF information

\begin{tabular}{lll}
\hline$q=3$ & $\breve{G}_{1}$ & $\breve{G}_{2}$ \\
\hline$M_{1}$ & $(\langle 1,0.959\rangle,\langle 0.798,0.733\rangle)$ & $(\langle 0.895,0.925\rangle,\langle 0.767,0.745\rangle)$ \\
$M_{2}$ & $(\langle 1,0.931\rangle,\langle 0.7,0.8172\rangle)$ & $(\langle 1,0.897\rangle,\langle 0.8,0.719\rangle)$ \\
$M_{3}$ & $(\langle 0.864,0.909\rangle,\langle 0.672,0.845\rangle)$ & $(\langle 1,0.881\rangle,\langle 0.769,0.763\rangle)$ \\
$M_{4}$ & $(\langle 1,0.966\rangle,\langle 0.803,0.734\rangle)$ & $(\langle 1,0.934\rangle,\langle 0.725,0.806\rangle)$ \\
\hline & $\breve{G}_{3}$ & $\breve{G}_{4}$ \\
\hline$M_{1}$ & $(\langle 1,0.92\rangle,\langle 0.7,0.812\rangle)$ & $(\langle 0.882,0.949\rangle,\langle 0.836,0.691\rangle)$ \\
$M_{2}$ & $(\langle 0.844,0.98\rangle,\langle 0.78,0.724\rangle)$ & $(\langle 1,0.912\rangle,\langle 0.747,0.758\rangle)$ \\
$M_{3}$ & $(\langle 0.925,0.858\rangle,\langle 0.747,0.77\rangle)$ & $(\langle 0.893,0.987\rangle,\langle 0.845,0.663\rangle)$ \\
$M_{4}$ & $(\langle 1,0.916\rangle,\langle 0.8,0.7\rangle)$ & $(\langle 0.928,0.873\rangle,\langle 0.742,0.782\rangle)$ \\
\hline & $\breve{G}_{5}$ & \\
\hline$M_{1}$ & $(\langle 0.862,0.9\rangle,\langle 0.7,0.837\rangle)$ & \\
$M_{2}$ & $(\langle 1,0.9\rangle,\langle 0.6,0.9\rangle)$ & \\
$M_{3}$ & $(\langle 0.88,0.96\rangle,\langle 0.8,0.7\rangle)$ & \\
$M_{4}$ & $(\langle 1,0.974\rangle,\langle 0.84,0.706\rangle)$ & \\
\hline & & \\
\end{tabular}


Table 11 q-RLDFWA

$(\langle 1,0.93612\rangle,\langle 0.774809,0.75320653\rangle)$

$(\langle 1,0.923948\rangle,\langle 0.746725,0.772728625\rangle)$

$(\langle 1,0.907903\rangle,\langle 0.758513,0.767757496\rangle)$

$(\langle 1,0.935836\rangle,\langle 0.7818,0.750527441\rangle)$

Table 12 q-RLDFOWA

$(\langle 1,0.933521\rangle,\langle 0.777614,0.749313229\rangle)$

$(\langle 1,0.910213\rangle,\langle 0.745997,0.775714396\rangle)$

$(\langle 1,0.914288\rangle,\langle 0.769304,0.75636881\rangle)$

$(\langle 1,0.939697\rangle,\langle 0.784026,0.74633646\rangle)$

Table 13 q-RLDFHWA

\begin{tabular}{ll}
\hline$\sigma_{\hbar 1}$ & $(\langle 1,0.944706\rangle,\langle 0.76158,0.767350765\rangle)$ \\
$\sigma_{\hbar 2}$ & $(\langle 1,0.929961\rangle,\langle 0.736473,0.784808601\rangle)$ \\
$\sigma_{\hbar 3}$ & $(\langle 1,0.908763\rangle,\langle 0.729841,0.797802586\rangle)$ \\
$\sigma_{\hbar 4}$ & $(\langle 1,0.947738\rangle,\langle 0.76717,0.765998282\rangle)$ \\
\hline
\end{tabular}

Algorithm-2 Algorithm based on q-RLDFWG Aggregation operator

Algorithm 2 is same as algorithm 1 and having the same steps as described in 1st algorithm, but here in algorithm 2 we apply q-RLDFWG Aggregation operator by using eq 7.4, 7.5 and 7.6. Just replace q-RLDFWA by q-RLDFWG in 1st algorithm then it become Algorithm 2nd.

\section{Algorithm-2 Solution:}

We choose our preceding input data shown in Tables 7, 8 and 9 for this algorithm. Using step 4 in above Algorithm, we find the final set of weights, so we get $\ell=(0.4,0.35,0.25)^{T}$. It is clear from final weight vector that $\sum_{\zeta=1}^{3} \ell$

Now we use q-RLDFWG, q-RLDFOWG, and q-RLDFHWG operators to calculate the collective (combine) q-RLDF information, given in Table 16.

$\Rightarrow$ Let we want to find q-RLDFOWG, then same process can be apply as we did in q-RLDFWG but in current method we first find order of each attributes of three experts individually by using SF, QSF, ESF. The main factor of the OWG operator is the reordering step; it first reorders all the given input data (attributes) in descending order and then weights these ordered input data (attributes), and finally aggregates (combines) all these ordered weighted attributes into a collective one. We repeat same steps of this Algorithm.

$\Rightarrow$ Again, we construct the weight vectors by using step 4 of this algorithm, corresponding to the collective q-RLDFWG decision matrix. Let the five weight vectors for Table 16 of q-RLDF data are given as

$$
\begin{aligned}
& { }^{1} \ell=(0.3,0.25,0.2,0.15,0.1)^{T} \\
& { }^{2} \ell=(0.25,0.35,0.2,0.1,0.1)^{T} \\
& { }^{3} \ell=(0.35,0.25,0.15,0.15,0.1)^{T} \\
& { }^{4} \ell=(0.3,0.3,0.2,0.1,0.1)^{T} \\
& { }^{5} \ell=(0.4,0.2,0.1,0.2,0.1)^{T}
\end{aligned}
$$

We calculate these weights by using step 4, So we obtained the final weight vectors given as

$\ell=(0.32,0.27,0.17,0.14,0.1)^{T}$. It is clear from final weight vectors that $\sum_{\zeta=1}^{5} \ell \zeta=1$.

$\Rightarrow$ We again repeat above step and aggregate Table 16 alternative wise by using eq 7.4, 7.5 and 7.6 with weights $\ell=(0.32,0.27,0.17,0.14,0.1)^{T}$, So we get q-RLDFWG, q-RLDFOWG, q-RLDFHWG decision matrix $D$ as described in Tables 17, 18 and 19.

$\Rightarrow$ After that we determine the value of its score by using SF, QSF and ESF via definition (16-22), we get Table 20;

$\Rightarrow$ Next we ranked (Table 20) the attributes of q-RLDFWG, q-RLDFOWG and q-RLDFHWG, we get the final result shown in Table 21;

$\Rightarrow$ From this we conclude that; $M_{4}$ is chose the best alternative (method) for Corona virus prevention and control.

\section{Discussion and comparison analysis}

Within this section we have a comparison of the suggested six q-RLDF aggregation operators with existing operators presented in (Riaz and Hashmi 2019), showing the strength to handle real-life decision-making problems (DMPs) with uncertainty. The impressive point of this concept is that, it covers the valuation spaces of IFSs, PyFSs, q-ROFSs and LDFSs because of qth power of reference parameters. We can see the ranking results of four alternatives by using existing approaches and proposed concept given in Tables 22 and 23. Table 23 shows that, health expert opinion on the prevention and control of Corona virus made by proposed method is same as the existing methods, which is expressive in itself and approves the reliability and validity of the suggested method and show that medical support is mandatory in the prevention of viral disease (COVID19). People should be follows doctor advice like uses of surgical mask at all times etc. to prevent spread of corona virus. There is a slightly difference between the ranking results derived from the proposed approach and existing approach but the best and first choice is same all in the both methods. The results of the comparison are seen in Tables 23, 24 below. Now we will share the validity and versatility of the established method for handling different outputs and inputs. 
Table 14 q-RLDFWA Score values

\begin{tabular}{lllll}
\hline S.F $(\kappa)$ & & & & \\
\hline$q=3$ & $\kappa_{\sigma_{h 1}}$ & $\kappa_{\sigma_{h 2}}$ & $\kappa_{\sigma_{h 3}}$ & $\kappa_{\sigma_{h 4}}$ \\
\hline q-RLDFWA & 0.051 & 0.016 & 0.038 & 0.06 \\
q-RLDFOWA & 0.058 & 0.019 & 0.054 & 0.063 \\
q-RLDFHWA & 0.023 & -0.006 & -0.013 & 0.027 \\
\hline Q.S.F( $)$ & & & & \\
\hline$q=3$ & $\varpi_{\sigma_{h 1}}$ & $\varpi_{\sigma 2}$ & $\varpi_{\sigma_{h 3}}$ & $\varpi_{\sigma_{h 4}}$ \\
\hline q-RLDFWA & 0.079 & 0.053 & 0.081 & 0.087 \\
q-RLDFOWA & 0.086 & 0.063 & 0.092 & 0.092 \\
q-RLDFHWA & 0.049 & 0.031 & 0.034 & 0.052 \\
\hline E.S.F(F) & & & & \\
\hline$q=3$ & $F_{\sigma 1}$ & $F_{\sigma 2}$ & $F_{\sigma_{h 3}}$ & $F_{\sigma_{h 4}}$ \\
\hline q-RLDFWA & 0.525 & 0.508 & 0.519 & 0.53 \\
q-RLDFOWA & 0.529 & 0.51 & 0.527 & 0.532 \\
q-RLDFHWA & 0.511 & 0.497 & 0.493 & 0.514 \\
\hline
\end{tabular}

Table 15 Ranking of q-RLDFWA operator

\begin{tabular}{lll}
\hline$q=3$ & S.F $(\kappa)$ & Q.S.F $(\varpi)$ \\
\hline q-RLDFWA & $M_{4}>M_{1}>M_{3}>M_{2}$ & $M_{4}>M_{3}>M_{1}>M_{2}$ \\
q-RLDFOWA & $M_{4}>M_{1}>M_{3}>M_{2}$ & $M_{4}=M_{3}>M_{1}>M_{2}$ \\
q-RLDFHWA & $M_{4}>M_{1}>M_{2}>M_{3}$ & $M_{4}>M_{1}>M_{3}>M_{2}$ \\
\hline$q=3$ & E.S.F $(F)$ & \\
\hline q-RLDFWA & $M_{4}>M_{1}>M_{3}>M_{2}$ & \\
q-RLDFOWA & $M_{4}>M_{1}>M_{3}>M_{2}$ & \\
q-RLDFHWA & $M_{4}>M_{1}>M_{2}>M_{3}$ & \\
\hline
\end{tabular}

Table 16 The collective q-RLDFWG decision matrix DM

\begin{tabular}{lll}
\hline$q=3$ & $\breve{G}_{1}$ & $\breve{G}_{2}$ \\
\hline$M_{1}$ & $(\langle 0.891,1\rangle,\langle 0.782,0.742\rangle)$ & $(\langle 0.895,1\rangle,\langle 0.744,0.78\rangle)$ \\
$M_{2}$ & $(\langle 0.996,1\rangle,\langle 0.7,0.82\rangle)$ & $(\langle 0.979,1\rangle,\langle 0.8,0.724\rangle)$ \\
$M_{3}$ & $(\langle 0.857,1\rangle,\langle 0.645,0.872\rangle)$ & $(\langle 0.925,1\rangle,\langle 0.749,0.771\rangle)$ \\
$M_{4}$ & $(\langle 0.934,1\rangle,\langle 0.745,0.789\rangle)$ & $(\langle 0.964,1\rangle,\langle 0.706,0.831\rangle)$ \\
\hline & $\breve{G}_{3}$ & $\breve{G}_{4}$ \\
\hline$M_{1}$ & $(\langle 0.929,1\rangle,\langle 0.7,0.814\rangle)$ & $(\langle 0.88,1\rangle,\langle 0.811,0.726\rangle)$ \\
$M_{2}$ & $(\langle 0.84,1\rangle,\langle 0.774,0.731\rangle)$ & $(\langle 0.924,1\rangle,\langle 0.738,0.767\rangle)$ \\
$M_{3}$ & $(\langle 0.906,0.861\rangle,\langle 0.738,0.784\rangle)$ & $(\langle 0.892,1\rangle,\langle 0.834,0.671\rangle)$ \\
$M_{4}$ & $(\langle 0.962,1\rangle,\langle 0.8,0.7\rangle)$ & $(\langle 0.909,0.896\rangle,\langle 0.733,0.798\rangle)$ \\
\hline & $\breve{G}_{5}$ & \\
\hline$M_{1}$ & $(\langle 0.835,0.914\rangle,\langle 0.7,0.839\rangle)$ & \\
$M_{2}$ & $(\langle 1,0.9\rangle,\langle 0.6,0.9\rangle)$ & \\
$M_{3}$ & $(\langle 0.88,0.96\rangle,\langle 0.8,0.7\rangle)$ & \\
$M_{4}$ & $(\langle 0.924,1\rangle,\langle 0.795,0.761\rangle)$ & \\
\hline
\end{tabular}


Table 17 q-RLDFWG

$(\langle 0.891044,1\rangle,\langle 0.75285,0.776387327\rangle)$

$(\langle 0.953575,1\rangle,\langle 0.732323,0.791300941\rangle)$

$(\langle 0.890395,1\rangle,\langle 0.727791,0.80009093\rangle)$

$(\langle 0.942005,1\rangle,\langle 0.746266,0.78920172\rangle)$

Table 18 q-RLDFOWG

$(\langle 0.881497,1\rangle,\langle 0.756074,0.772503599\rangle)$

$(\langle 0.953579,1\rangle,\langle 0.731639,0.793467497\rangle)$

$(\langle 0.883908,1\rangle,\langle 0.740457,0.787467067\rangle)$

$(\langle 0.941565,1\rangle,\langle 0.748525,0.785041962\rangle)$

Table 19 q-RLDFHWG

$(\langle 0.920206,1\rangle,\langle 0.819655,0.713933352\rangle)$

$(\langle 0.964281,1\rangle,\langle 0.799331,0.732012787\rangle)$

$(\langle 0.921256,1\rangle,\langle 0.817189,0.711341432\rangle)$

$(\langle 0.956696,1\rangle,\langle 0.82358,0.712128595\rangle)$

Validity and simplicity of the propose method: Our proposed method is applicable and appropriate for input data of all types. The model suggested is effective for addressing uncertainties. With the introduction of qth power of reference parameters, this approach covers the area of IFS, PFS, q-ROFS and LDFS. Adding qth power to parameters increases more membership and non-member space and changes the physical meaning of these parameters. We may use our method effectively in different circumstances, in present work we apply it for COVID-19 disease. The proposed q-RLDFS is clear and simple, and can be easily extended to various results.

The Influence of score function: We generalized and then implement the already introduced three types of score functions called SF, QSF and ESF ans also generalized associated accuracy functions (AFs). Each SF has its own observation and ordering procedures so afford that a little different effect. We can see from Tables 23 and 24 that SF, ESF and QSF rankings differ slightly from each other. Although it is important to keep in mind that the final result through both algorithms is nearly identical for all score functions.

Flexibility of aggregation with different inputs and outputs: This approach is much more flexible than others as qth power of reference parameters increases grade space and can differ depending on the situations in MADM methods. And it can be used conveniently for various input and output conditions.

Sensitivity analysis: One can observe the sensitivity analysis of the suggested models from Tables 15 and 21 . Both algorithms obtain identical results. The change in alternative rankings is due to differences in score functions but the optimal result is same. So it's obvious both algorithms are only sensitive to the functions of the score.

Superiority and comparison of proposed method with existing approaches: q-RLDFS has lots of space compared to IFSs, PFSs, q-ROFSs and LDFSs since q-RLDFS handles qth parameterizations. Riaz and Hashmi (2019) introduced LDFSs with addition of reference parameters but LDFSs have also some limitation on reference parameters and cannot deal with qth parameterization. We extended

Table 20 q-RLDFWG Score values

\begin{tabular}{|c|c|c|c|c|}
\hline \multirow[b]{2}{*}{$q=3$} & \multicolumn{4}{|l|}{$\mathrm{S} . \mathrm{F}(\kappa)$} \\
\hline & $\kappa_{\sigma_{h 1}}$ & $\kappa_{\sigma_{h 2}}$ & $\kappa_{\sigma_{h 3}}$ & $\kappa_{\sigma_{h 4}}$ \\
\hline$q-R L D F W G$ & -0.08 & -0.074 & -0.12 & -0.066 \\
\hline$q-R L D F O W G$ & -0.07 & -0.08 & -0.1 & -0.06 \\
\hline \multirow[t]{2}{*}{$q-R L D F H W G$} & 0.053 & 0.041 & 0.054 & 0.077 \\
\hline & \multicolumn{4}{|c|}{ E.S.F $(F)$} \\
\hline$q=3$ & $F_{\sigma 1}$ & $F_{\sigma 2}$ & $F_{\sigma_{h 3}}$ & $F_{\sigma_{h 4}}$ \\
\hline$q-R L D F W G$ & 0.462 & 0.463 & 0.441 & 0.467 \\
\hline$q-R L D F O W G$ & 0.465 & 0.461 & 0.45 & 0.469 \\
\hline \multirow[t]{2}{*}{$q-R L D F H W G$} & 0.527 & 0.521 & 0.527 & 0.539 \\
\hline & \multicolumn{4}{|c|}{ Q.S.F $(\varpi)$} \\
\hline$q=3$ & $\varpi_{\sigma_{h 1}}$ & $\varpi_{\sigma 2}$ & $\varpi_{\sigma_{h 3}}$ & $\varpi_{\sigma_{h 4}}$ \\
\hline$q-R L D F W G$ & -0.12 & -0.0909 & -0.16 & -0.0907 \\
\hline$q-R L D F O W G$ & -0.12 & -0.093 & -0.15 & -0.085 \\
\hline$q-R L D F H W G$ & 0.009 & 0.018 & 0.008 & 0.048 \\
\hline
\end{tabular}


Table 21 Ranking of q-RLDFWG operator

\begin{tabular}{lll}
\hline$q=3$ & S.F $(\kappa)$ & Q.S.F $(\varpi)$ \\
\hline q-RLDFWG & $M_{4}>M_{2}>M_{1}>M_{2}$ & $M_{4}>M_{2}>M_{1}>M_{3}$ \\
q-RLDFOWG & $M_{4}>M_{1}>M_{2}>M_{3}$ & $M_{4}>M_{2}>M_{1}>M_{2}$ \\
q-RLDFHWG & $M_{4}>M_{3}>M_{1}>M_{2}$ & $M_{4}>M_{2}>M_{1}>M_{3}$ \\
\hline$q=3$ & E.S.F $(F)$ & \\
\hline q-RLDFWG & $M_{4}>M_{2}>M_{1}>M_{3}$ & \\
q-RLDFOWG & $M_{4}>M_{1}>M_{2}>M_{3}$ & \\
q-RLDFHWG & $M_{4}>M_{1}=M_{3}>M_{2}$ & \\
\hline
\end{tabular}

the concept of LDFSs and proposed q-RLDFSs, which fill this research gap. Proposed method and MADM problems have a close relationship. In this method the qth power of reference parameters plays a major role. So compared to other approaches, we can get more accurate results with our q-RLDFS based method .

The ranking order of corona virus prevention and control alternatives are described in Table 25.

The column chart of score, Quadratic and Expectation score functions values based on q-RLDFWA aggregation operator is given in Table 22, Figs. 5, 6 and 7 respectiv ely.

\section{Conclusion}

In today's world, with increased human activities and massive economic development, emergency incidents happened more commonly, which directly threatened the life and property of people. Emergency decision-making (DM) performs a noticeable role in decreasing casualties and has gained much public interest (Fig. 8). In ordered to remove such restriction, Riaz and Hashmi (2019) introduced concept of LDFS in which they introduced the role of reference parameters, which hold the condition
$0 \leq(\alpha) \wp_{D(\hbar)}+(\beta) \Re_{D(\hbar)} \leq 1, \forall \hbar \in M$ with $0 \leq \alpha+\beta \leq 1$. But here again, the sum of reference parameters provided by DM may lager than one i.e. $\alpha+\beta>1$, which contradicts the constraint of LDFS. So LDFS did not achieved his goal related to reference parameters. It makes the MADM limited, and affects the optimum decision. In order to eliminate this contradiction, we introduce the novel idea of the q-RLDFS which is capable of dealing with these situations and to explain the concept of q-RLDFSs. In this manuscript, we developed the q-RLDF emergency DM problem based on the current outbreak of corona virus or COVID-19. The proposed method helped us that how to prevent from COVID19. Some extensions of FSs have been studied including IFSs, PFSs, q-ROFSs, and LDFSs. We've developed a new updated version of FS called q-RLDFS which is more powerful and flexible to manage uncertainties. The qth power of reference parameters notion can provide a more flexible and efficient framework for fuzzy system modeling and decision making under uncertainty.

To relate it to other established FSs extensions, we have presented the geometric and averaging properties of q-RLDFS. For comparison of q-RLDFNs, we adopted various SFs and AFs, and then generalized these functions and got an advanced scored function. We have extended the idea of q-RLDFS to q-RLDFWAA and q-RLDFWGA operators.

Table 22 Discussion on q-RLDF's comparative study with existing approaches

\begin{tabular}{|c|c|c|}
\hline Collections & Remarks & Parameterization \\
\hline FS (Zadeh 1965) & Unable to handle non-membership $\Re_{(\hbar)}$ & No \\
\hline IFS (Atanassov 1986) & cannot deal with the condition, $\wp_{(\hbar)}+\Re_{(\hbar)}>1$ & No \\
\hline PyFS (Yager 2013a, b) & cannot deal with the condition, $\wp_{(\hbar)}^{2}+\mathfrak{R}_{(\hbar)}^{2}>1$ & No \\
\hline q-ROFS (Yager 2016) & $\begin{array}{l}\text { Unable to deal with smaller "q" values with the condition, } \\
\wp_{(\hbar)}^{q}+\mathfrak{R}_{(\hbar)}^{q}>1 \text { and for } \wp_{(\hbar)}=1, \mathfrak{R}_{(\hbar)}=1\end{array}$ & No \\
\hline LDFS (Riaz 2019) & $\begin{array}{l}\text { This collection covers upon this situation, } 0 \leq(\alpha) \wp_{D(\hbar)}+(\beta) \mathfrak{R}_{D(\hbar)} \leq 1 \text {, and also works under the } \\
\text { influence of reference parameters }\langle\alpha, \beta\rangle \text {. So the only existing method is LDF operators which we } \\
\text { compare to our proposed method. }\end{array}$ & Yes \\
\hline
\end{tabular}


Table 23 Comparison analysis of q-RLDF with existing approach

\begin{tabular}{|c|c|c|c|c|}
\hline \multirow{2}{*}{$\begin{array}{l}q=3 \\
\text { Proposed method }\end{array}$} & \multicolumn{4}{|l|}{$\mathrm{S} . \mathrm{F}(\kappa)$} \\
\hline & $\kappa_{\sigma_{h 1}}$ & $\kappa_{\sigma_{h 2}}$ & $\kappa_{\sigma_{h 3}}$ & $\kappa_{\sigma_{h 4}}$ \\
\hline q-RLDFWA & 0.051 & 0.016 & 0.038 & 0.06 \\
\hline q-RLDFOWA & 0.058 & 0.019 & 0.054 & 0.063 \\
\hline q-RLDFHWA & 0.023 & -0.006 & -0.013 & 0.027 \\
\hline q-RLDFWG & -0.08 & -0.074 & -0.12 & -0.066 \\
\hline q-RLDFOWG & -0.07 & -0.08 & -0.1 & -0.06 \\
\hline q-RLDFHWG & 0.053 & 0.041 & 0.054 & 0.077 \\
\hline Existing method & $\kappa_{\sigma_{h 1}}$ & $\kappa_{\sigma_{h 2}}$ & $\kappa_{\sigma_{h 3}}$ & $\kappa_{\sigma_{h 4}}$ \\
\hline LDFWA (Riaz 2019) & 0.0403 & 0.0232 & 0.0378 & 0.0439 \\
\hline LDFOWA (Riaz 2019) & 0.045 & 0.0282 & 0.0461 & 0.0453 \\
\hline LDFWG (Riaz 2019) & -0.064 & -0.051 & -0.088 & -0.046 \\
\hline LDFOWG (Riaz 2019) & -0.061 & -0.052 & -0.078 & -0.043 \\
\hline$q=3$ & Q.S.F( $(\varpi)$ & & & \\
\hline Proposed method & $\varpi_{\sigma_{h 1}}$ & $\varpi_{\sigma 2}$ & $\varpi_{\sigma_{h 3}}$ & $\varpi_{\sigma_{h 4}}$ \\
\hline q-RLDFWA & 0.079 & 0.053 & 0.081 & 0.087 \\
\hline q-RLDFOWA & 0.086 & 0.063 & 0.092 & 0.092 \\
\hline q-RLDFHWA & 0.049 & 0.031 & 0.034 & 0.052 \\
\hline q-RLDFWG & -0.12 & -0.0909 & -0.16 & $\begin{array}{c}- \\
0.0907\end{array}$ \\
\hline q-RLDFOWG & -0.12 & -0.093 & -0.15 & -0.085 \\
\hline q-RLDFHWG & 0.009 & 0.018 & 0.008 & 0.048 \\
\hline Existing method & $\varpi_{\sigma_{h 1}}$ & $\varpi_{\sigma 2}$ & $\varpi_{\sigma_{h 3}}$ & $\varpi_{\sigma_{h 4}}$ \\
\hline LDFWA (Riaz 2019) & 0.0745 & 0.0507 & 0.0754 & 0.0802 \\
\hline LDFOWA (Riaz 2019) & 0.082 & 0.06 & 0.087 & 0.082 \\
\hline LDFWG (Riaz 2019) & -0.117 & -0.087 & -0.154 & $\begin{array}{r}- \\
0.083\end{array}$ \\
\hline LDFOWG (Riaz 2019) & -0.113 & -0.0895 & -0.14 & $\begin{array}{r}- \\
0.078\end{array}$ \\
\hline$q=3$ & E.S.F $(F)$ & & & \\
\hline Proposed method & $F_{\sigma 1}$ & $F_{\sigma 2}$ & $F_{\sigma_{h 3}}$ & $F_{\sigma_{h 4}}$ \\
\hline q-RLDFWA & 0.525 & 0.508 & 0.519 & 0.53 \\
\hline q-RLDFOWA & 0.529 & 0.51 & 0.527 & 0.532 \\
\hline q-RLDFHWA & 0.511 & 0.497 & 0.493 & 0.514 \\
\hline q-RLDFWG & 0.462 & 0.463 & 0.441 & 0.467 \\
\hline q-RLDFOWG & 0.465 & 0.461 & 0.45 & 0.469 \\
\hline q-RLDFHWG & 0.527 & 0.521 & 0.527 & 0.539 \\
\hline Existing method & $F_{\sigma 1}$ & $F_{\sigma 2}$ & $F_{\sigma_{h 3}}$ & $F_{\sigma_{h 4}}$ \\
\hline LDFWA (Riaz 2019) & 0.5201 & 0.5116 & 0.5189 & 0.522 \\
\hline LDFOWA (Riaz 2019) & 0.523 & 0.514 & 0.523 & 0.523 \\
\hline LDFWG (Riaz 2019) & 0.468 & 0.475 & 0.456 & 0.477 \\
\hline LDFOWG (Riaz 2019) & 0.4697 & 0.474 & 0.461 & 0.478 \\
\hline
\end{tabular}


Table 24 Overall ranking ordered of proposed and existing method

\begin{tabular}{|c|c|c|}
\hline Proposed Method & $\operatorname{S.F}(\kappa)$ & Q.S.F( $(\varpi)$ \\
\hline q-RLDFWA & $M_{4}>M_{1}>M_{3}>M_{2}$ & $M_{4}>M_{3}>M_{1}>M_{2}$ \\
\hline q-RLDFOWA & $M_{4}>M_{1}>M_{3}>M_{2}$ & $M_{4}=M_{3}>M_{1}>M_{2}$ \\
\hline q-RLDFHWA & $M_{4}>M_{1}>M_{2}>M_{3}$ & $M_{4}>M_{1}>M_{3}>M_{2}$ \\
\hline q-RLDFWG & $M_{4}>M_{2}>M_{1}>M_{2}$ & $M_{4}>M_{2}>M_{1}>M_{3}$ \\
\hline q-RLDFOWG & $M_{4}>M_{1}>M_{2}>M_{3}$ & $M_{4}>M_{2}>M_{1}>M_{2}$ \\
\hline q-RLDFHWG & $M_{4}>M_{3}>M_{1}>M_{2}$ & $M_{4}>M_{2}>M_{1}>M_{3}$ \\
\hline Existing Method & $\mathrm{S} . \mathrm{F}(\kappa)$ & Q.S.F( $(\varpi)$ \\
\hline LDFWA (Riaz 2019) & $M_{4}>M_{1}>M_{3}>M_{2}$ & $M_{4}>M_{3}>M_{1}>M_{2}$ \\
\hline LDFOWA (Riaz 2019) & $M_{3}>M_{4}>M_{1}>M_{2}$ & $M_{3}>M_{4}=M_{1}>M_{2}$ \\
\hline LDFWG (Riaz 2019) & $M_{4}>M_{2}>M_{1}>M_{3}$ & $M_{4}>M_{2}>M_{1}>M_{3}$ \\
\hline LDFOWG (Riaz 2019) & $M_{4}>M_{2}>M_{1}>M_{3}$ & $M_{4}>M_{2}>M_{1}>M_{3}$ \\
\hline Proposed Method & E.S.F $(F)$ & \\
\hline q-RLDFWA & $M_{4}>M_{1}>M_{3}>M_{2}$ & \\
\hline q-RLDFOWA & $M_{4}>M_{1}>M_{3}>M_{2}$ & \\
\hline q-RLDFHWA & $M_{4}>M_{1}>M_{2}>M_{3}$ & \\
\hline q-RLDFWG & $M_{4}>M_{2}>M_{1}>M_{3}$ & \\
\hline q-RLDFOWG & $M_{4}>M_{1}>M_{2}>M_{3}$ & \\
\hline q-RLDFHWG & $M_{4}>M_{1}=M_{3}>M_{2}$ & \\
\hline Existing Method & E.S.F $(F)$ & \\
\hline LDFWA & $M_{4}>M_{1}>M_{3}>M_{2}$ & \\
\hline LDFOWA & $M_{4}=M_{3}=M_{1}>M_{2}$ & \\
\hline LDFWG [?] & $M_{4}>M_{2}>M_{1}>M_{3}$ & \\
\hline LDFOWG [?] & $M_{4}>M_{2}>M_{1}>M_{3}$ & \\
\hline
\end{tabular}

Table 25 COVID-19 preventive alternatives ordered

\begin{tabular}{lll}
\hline Ranking & Notation & Alternatives \\
\hline 1 & $M_{4}$ & Medical support \\
2 & $M_{1}$ & Online first-Aid course \\
3 & $M_{3}$ & Local government,s decision \\
4 & $M_{2}$ & Vaccination \\
\hline
\end{tabular}

Furthermore, the decision frameworks presented in this study can be straightforwardly applied to real-world group decision making problems including both q-RLDFWA and q-RLDFWG data. With the guidance of a case study for the prevention and control of corona virus, we provided an implementation of the proposed MADM methods. Finally we compared proposed method with some existing method. The obtain results indicated the benefits and applicability of the proposed technique.

In future work, we will expand this work to other generalized theories of fuzzy environment, which is interval-valued intuitionistic fuzzy sets (IVIFSs), cubic fuzzy sets (CFSs), hesitant fuzzy sets (HFSs), Hamacher operators, Dombi operators, etc. And also will focus on applying the proposed method to solve practical MAGDM problems like emerging technology, uncertain decision-making, project installation, site selection etc. 


\begin{tabular}{|c|c|c|}
\hline Collections & Remarks & Parameterization \\
\hline FS [15] & Unable to handle non-membership $\Re_{(\hbar)}$ & $\mathrm{NO}$ \\
\hline Intuitionistics fuzzy set [14] & cannot deal with the condition, $\wp_{(\hbar)}+\Re_{(\hbar)}>1$ & $\mathrm{NO}$ \\
\hline Pythagorean fuzzy set $[32,33]$ & cannot deal with the condition, $\wp_{(\hbar)}^{2}+\Re_{(\hbar)}^{2}>1$ & NO \\
\hline q-ROFS [45] & $\begin{array}{l}\text { Unable to deal with smaller " } q \text { " values } \\
\text { with the condition, } \\
\wp_{(\hbar)}^{q}+\Re_{(\hbar)}^{q}>1 \text { and for } \wp_{(\hbar)}=1, \Re_{(\hbar)}=1\end{array}$ & $\mathrm{NO}$ \\
\hline LDFS [67] & $\begin{array}{l}\text { This collection covers upon this situation, } \\
0 \leq(\alpha) \wp_{D(\hbar)}+(\beta) \Re_{D(\hbar)} \leq 1 \\
\text { and also works under the influence of } \\
\text { reference parameters } \\
\langle\alpha, \beta\rangle \text {. So the only existing method } \\
\text { is LDF operators } \\
\text { which we compare to our } \\
\text { proposed method. }\end{array}$ & YES \\
\hline
\end{tabular}

Fig. 5 Alternative ranking under COVID-19 situation (q-RLDFWA)

Fig. 6 Alternative Quadratic Score Ranking under COVID19 situation (q-RLDFWA)

Fig. 7 Alternative Expectation Score Ranking under COVID19 situation (q-RLDFWA)

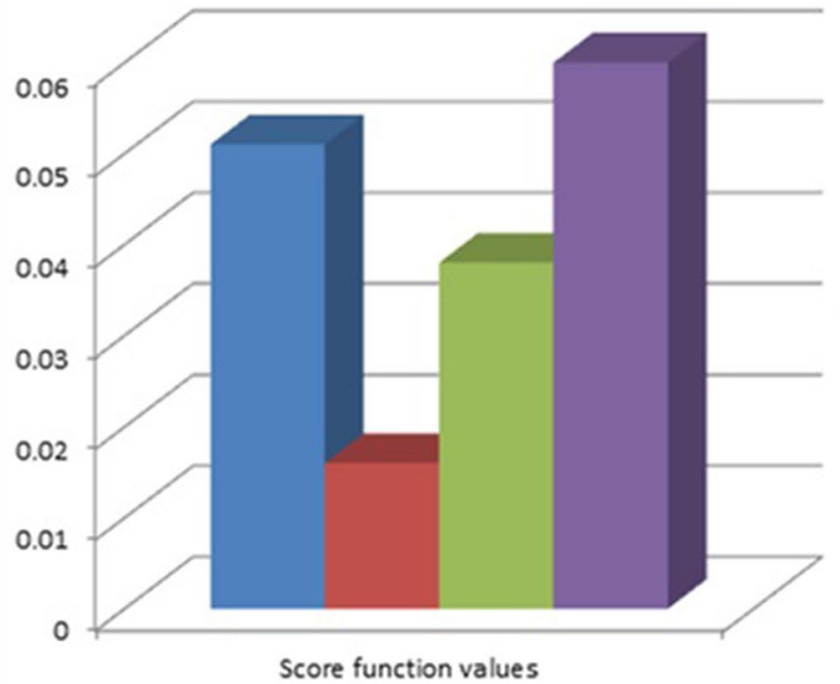

an On line first-Aid course

vaccination

in Local govemment, s decision

- Me dical support

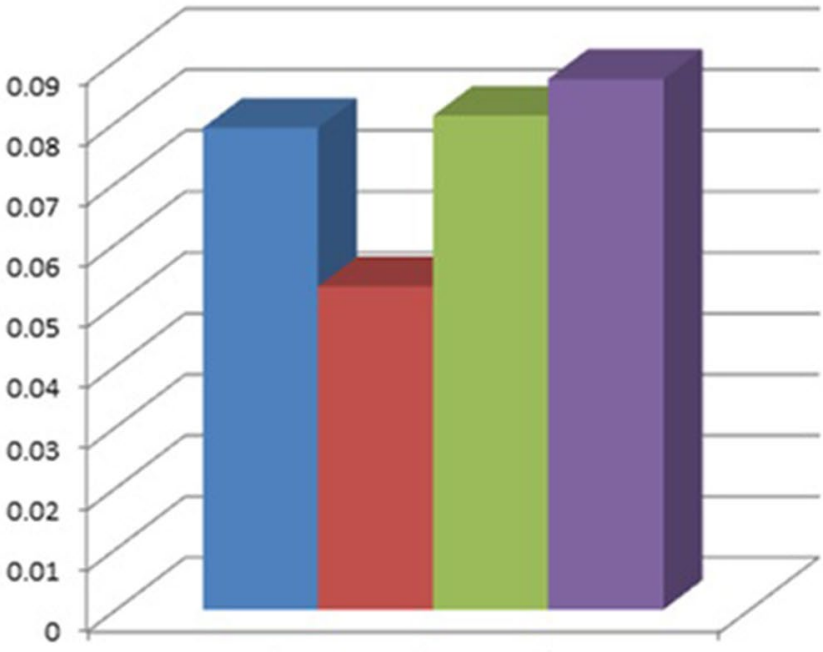

a Online first-Aid course

v Vaccination

in Local government, $\mathrm{s}$ decision

घMe dical support 
Fig. 8 Expectation score function

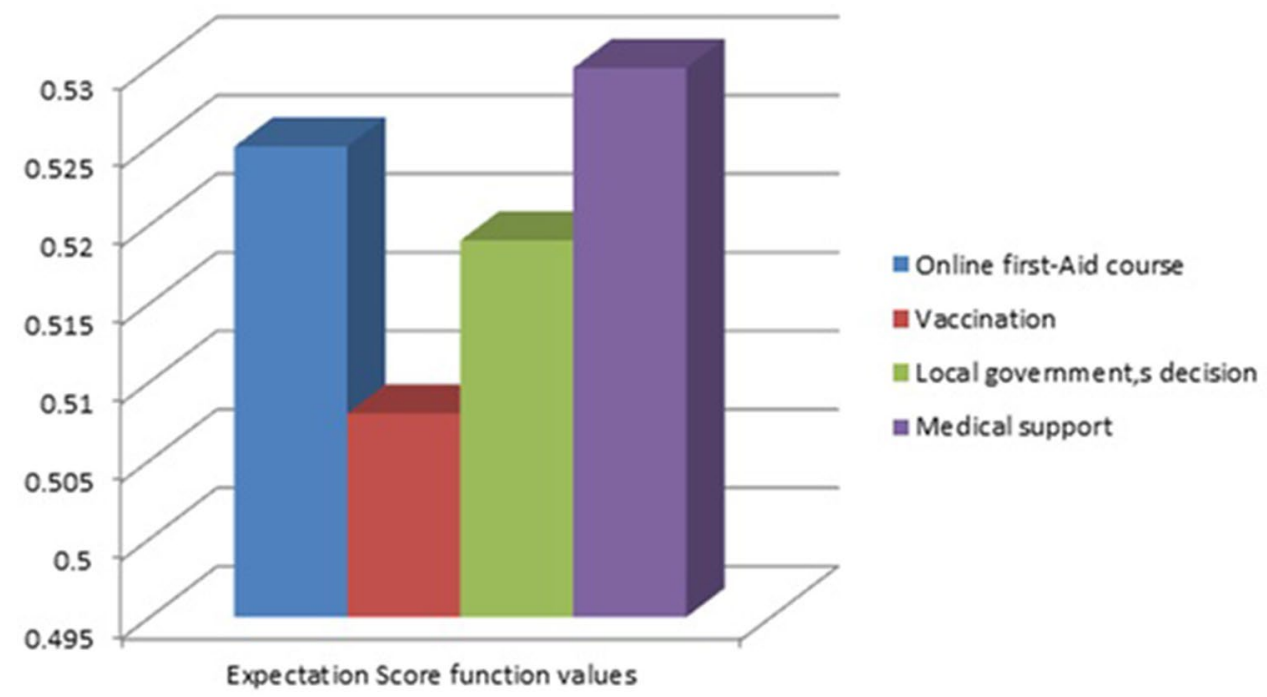

Acknowledgements This work was supported by the Deanship of Scientific Research (DSR), King Abdulaziz University, Jeddah, under grant No. (GCV19-10-1441). The authors, therefore, gratefully acknowledge DSR technical and financial support.

\section{References}

Ali MI (2018) Another view on q-rung orthopair fuzzy sets. Int J Intell Syst 33(11):2139-2153

Ashraf S, Abdullah S (2020) Emergency decision support modeling for COVID-19 based on spherical fuzzy information. Int J Intell Syst 35(11):1601-1645

Ashraf S, Abdullah S, Almagrabi AO (2020a) A new emergency response of spherical intelligent fuzzy decision process to diagnose of COVID19. Soft Comput. https://doi.org/10.1007/ s00500-020-05287-8

Ashraf S, Abdullah S, Khan S (2020b) Fuzzy decision support modeling for internet finance soft power evaluation based on sine trigonometric Pythagorean fuzzy information. J Ambient Intell Hum Comput. https://doi.org/10.1007/s12652-020-02471-4

Atanassov KT (1986) Intuitionistic fuzzy sets. Fuzzy Sets Syst 20(1):87-96

Atanassov K (1989) Geometrical interpretation of the elements of the intuitionistic fuzzy objects. Preprint IM-MFAIS-1-89, Sofia

Atanassov K, Gargov G (1989) Interval valued intuitionistic fuzzy sets. Fuzzy Sets Syst 31(3):343-349

Atanassov KT, Session VIS (1984) Sofia. Sgurev, V (Ed.). Central Sci. and Techn. Library, Bulg. Academy of Sciences (June 1983 )

Baharoon S, Memish ZA (2019) MERS-CoV as an emerging respiratory illness: a review of prevention methods. Travel Med Infect Dis 32:101520

Bai K, Zhu X, Wang J, Zhang R (2018) Some partitioned Maclaurin symmetric mean based on q-rung orthopair fuzzy information for dealing with multi-attribute group decision making. Symmetry 10(9):383

Batool B, Ahmad M, Abdullah S, Ashraf S, Chinram R (2020) Entropy based pythagorean probabilistic hesitant fuzzy Decision making technique and its application for Fog-Haze factor assessment problem. Entropy 22(3):318

Chan JFW, Yuan S, Kok KH, To KKW, Chu H, Yang J, Xing F, Liu J, Yip CCY, Poon RWS, Tsoi HW (2020) A familial cluster of pneumonia associated with the 2019 novel coronavirus indicating person-to-person transmission: a study of a family cluster. Lancet 395(10223):514-523

Chen SM, Tan JM (1994) Handling multicriteria fuzzy decisionmaking problems based on vague set theory. Fuzzy Sets Syst 67(2):163-172

Coronavirus COVID-19 global cases by the center for systems science and engineering (CSSE) at Johns Hopkins University (JHU). ArcGIS. Johns Hopkins CSSE. 2020. Retrieved 2020-04-07

Du WS (2019a) Research on arithmetic operations over generalized orthopair fuzzy sets. Int J Intell Syst 34(5):709-732

Du WS (2019b) Correlation and correlation coefficient of generalized orthopair fuzzy sets. Int J Intell Syst 34(4):564-583

Gao J, Liang Z, Shang J, Xu Z (2018) Continuities, derivatives, and differentials of $\backslash \$ \mathrm{q} \backslash \$$-rung orthopair fuzzy functions. IEEE Trans Fuzzy Syst 27(8):1687-1699

Garg H (2016a) Some series of intuitionistic fuzzy interactive averaging aggregation operators. SpringerPlus 5(1):999

Garg H (2016b) A new generalized improved score function of intervalvalued intuitionistic fuzzy sets and applications in expert systems. Appl Soft Comput 38:988-999

Garg H (2016c) A new generalized Pythagorean fuzzy information aggregation using Einstein operations and its application to decision making. Int J Intell Syst 31(9):886-920

Garg H (2017) Generalized Pythagorean fuzzy geometric aggregation operators using Einstein t-norm and t-conorm for multicriteria decision-making process. Int J Intell Syst 32(6):597-630

Garg H (2018a) Some methods for strategic decision-making problems with immediate probabilities in Pythagorean fuzzy environment. Int J Intell Syst 33(4):687-712

Garg H (2018b) Linguistic Pythagorean fuzzy sets and its applications in multiattribute decision-making process. Int J Intell Syst 33(6):1234-1263

Garg H (2018c) A linear programming method based on an improved score function for interval-valued Pythagorean fuzzy numbers and its application to decision-making. Int $\mathrm{J}$ Uncertain Fuzziness Knowl Based Syst 26(01):67-80

Garg H (2018d) New exponential operational laws and their aggregation operators for interval-valued Pythagorean fuzzy multicriteria decision-making. Int J Intell Syst 33(3):653-683

Garg H (2019a) New logarithmic operational laws and their aggregation operators for Pythagorean fuzzy set and their applications. Int J Intell Syst 34(1):82-106 
Garg H (2019b) New logarithmic operational laws and their aggregation operators for Pythagorean fuzzy set and their applications. Int J Intell Syst 34(1):82-106

Gou X, Xu Z (2017) Exponential operations for intuitionistic fuzzy numbers and interval numbers in multi-attribute decision making. Fuzzy Optim Decis Mak 16(2):183-204

Hämäläinen RP, Lindstedt MR, Sinkko K (2000) Multiattribute risk analysis in nuclear emergency management. Risk Anal 20(4):455-468

Herrera F, Martínez L (2001) A model based on linguistic 2-tuples for dealing with multigranular hierarchical linguistic contexts in multi-expert decision-making. IEEE Trans Syst Man Cybern Part B (Cybernetics) 31(2):227-234

Kahneman D, Tversky A (1979) Prospect theory: an analysis of decision under risk. Econometrica 47(2):263-292

Khan MJ, Kumam P, Liu P, Kumam W, Ashraf S (2019a) A novel approach to generalized intuitionistic fuzzy soft sets and its application in decision support system. Mathematics 7(8):742

Khan AA, Ashraf S, Abdullah S, Qiyas M, Luo J, Khan SU (2019b) Pythagorean fuzzy Dombi aggregation operators and their application in decision support system. Symmetry 11(3):383

Khan MJ, Kumam P, Liu P, Kumam W (2020a) Another view on generalized interval valued intuitionistic fuzzy soft set and its applications in decision support system. J Intell Fuzzy Syst 1:1-15

Khan MA, Ashraf S, Abdullah S, Ghani F (2020b) Applications of probabilistic hesitant fuzzy rough set in decision support system. Soft Comput 24:16759-16774

Khan MJ, Kumam P, Alreshidi NA, Shaheen N, Kumam W, Shah Z, Thounthong P (2020c) The renewable energy source selection by remoteness index-based VIKOR method for generalized intuitionistic fuzzy soft sets. Symmetry 12(6):977

Liu P, Liu J (2018) Some q-rung orthopai fuzzy Bonferroni mean operators and their application to multi-attribute group decision making. Int J Intell Syst 33(2):315-347

Liu P, Wang P (2018a) Some q-rung orthopair fuzzy aggregation operators and their applications to multiple-attribute decision making. Int J Intell Syst 33(2):259-280

Liu P, Wang P (2018b) Multiple-attribute decision-making based on Archimedean Bonferroni Operators of q-rung orthopair fuzzy numbers. IEEE Trans Fuzzy Syst 27(5):834-848

Liu P, Jin F, Zhang X, Su Y, Wang M (2011) Research on the multiattribute decision-making under risk with interval probability based on prospect theory and the uncertain linguistic variables. Knowl Based Syst 24(4):554-561

Liu Y, Fan ZP, Zhang Y (2014) Risk decision analysis in emergency response: a method based on cumulative prospect theory. Comput Oper Res 42:75-82

Liu X, Wang Z, Zhang S (2018a) A new methodology for hesitant fuzzy emergency decision making with unknown weight information. Complexity. https://doi.org/10.1155/2018/5145348

Liu P, Chen SM, Wang P (2018b) Multiple-attribute group decision-making based on q-rung orthopair fuzzy power maclaurin symmetric mean operators. IEEE Trans Syst Man Cybern Syst 50(10):3741-56

Liu Z, Wang S, Liu P (2018c) Multiple attribute group decision making based on q-rung orthopair fuzzy Heronian mean operators. Int J Intell Syst 33(12):2341-2363

Ma Z, Xu Z (2016) Symmetric Pythagorean fuzzy weighted geometric/averaging operators and their application in multicriteria decision-making problems. Int J Intell Syst 31(12):1198-1219

Newey BySarah, White J, Cameron-Chileshe B, Newey B, Gartner B, Gulland B, Roberts B, Louloudis B (2020) Coronavirus outbreak declared a pandemic: what does it mean, and does it change anything?

Peng X, Dai J (2019) Research on the assessment of classroom teaching quality with q-rung orthopair fuzzy information based on multiparametric similarity measure and combinative distancebased assessment. Int J Intell Syst 34(7):1588-1630

Peng X, Garg H (2019) Multiparametric similarity measures on Pythagorean fuzzy sets with applications to pattern recognition. Appl Intell 49(12):4058-4096

Peng X, Dai J, Garg H (2018) Exponential operation and aggregation operator for q-rung orthopair fuzzy set and their decisionmaking method with a new score function. Int J Intell Syst 33(11):2255-2282

Quiggin J (1991) Comparative statics for rank-dependent expected utility theory. J Risk Uncertain 4(4):339-350

Ren P, Xu Z, Hao Z (2017) Hesitant fuzzy thermodynamic method for emergency decision making based on prospect theory. IEEE Tran Cybern 47(9):2531-2543

Riaz M, Hashmi MR (2019) Linear Diophantine fuzzy set and its applications towards multi-attribute decision-making problems. J Intell Fuzzy Syst 37(4):5417-5439

Schmidt U, Starmer C, Sugden R, (2008) Risk aversion in cumulative prospect theory. Manag Sci 54(1):208-216

Tversky A, Kahneman D (1992) Advances in prospect theory: cumulative representation of uncertainty. J Risk Uncertain 5(4):297-323

Wang R, Li Y (2018) A novel approach for green supplier selection under a q-rung orthopair fuzzy environment. Symmetry 10(12):687

Wang L, Li N (2020) Pythagorean fuzzy interaction power Bonferroni mean aggregation operators in multiple attribute decision making. Int J Intell Syst 35(1):150-183

Wang Z, Li KW, Wang W (2009) An approach to multiattribute decision making with interval-valued intuitionistic fuzzy assessments and incomplete weights. Inf Sci 179(17):3026-3040

Wang L, Zhang ZX, Wang YM (2015) A prospect theory-based interval dynamic reference point method for emergency decision making. Exp Syst Appl 42(23):9379-9388

Wang P, Wang J, Wei G, Wei C (2019) Similarity measures of q-rung orthopair fuzzy sets based on cosine function and their applications. Mathematics 7(4):340

Wei G, Gao H, Wei Y (2018) Some q-rung orthopair fuzzy Heronian mean operators in multiple attribute decision making. Int J Intell Syst 33(7):1426-1458

Wei G, Wei C, Wang J, Gao H, Wei Y (2019) Some q-rung orthopair fuzzy maclaurin symmetric mean operators and their applications to potential evaluation of emerging technology commercialization. Int J Intell Syst 34(1):50-81

World Health Organization (2020a) Novel Coronavirus (2019-nCoV) Situation report-5, 25 January 2020. Switzerland, Geneva

World Health Organization, (2020b) Coronavirus disease 2019 (COVID-19) : situation report, 49

World Health Organization, (2020c) Global surveillance for COVID-19 disease caused by human infection with the 2019 novel coronavirus, Interim guidance, 27 February 2020

Wu Z, McGoogan JM (2020) Characteristics of and important lessons from the coronavirus disease 2019 (COVID-19) outbreak in China: summary of a report of 72314 cases from the Chinese Center for Disease Control and Prevention. Jama 323(13):1239-42

Xing Y, Zhang R, Zhou Z, Wang J (2019) Some q-rung orthopair fuzzy point weighted aggregation operators for multi-attribute decision making. Soft Comput 23(22):11627-11649

$\mathrm{Xu} \mathrm{Z} \mathrm{(2005)} \mathrm{An} \mathrm{overview} \mathrm{of} \mathrm{methods} \mathrm{for} \mathrm{determining} \mathrm{OWA} \mathrm{weights.}$ Int J Intell Syst 20(8):843-865

$\mathrm{Xu} \mathrm{Z}$, Yager RR (2006) Some geometric aggregation operators based on intuitionistic fuzzy sets. Int J Gener Syst 35(4):417-433

Yager RR (2013a) June. Pythagorean fuzzy subsets. In 2013 joint IFSA world congress and NAFIPS annual meeting (IFSA/NAFIPS) (pp. 57-61). IEEE 
Yager RR (2013b) Pythagorean membership grades in multicriteria decision making. IEEE Trans Fuzzy Syst 22(4):958-965

Yager RR (2016) Generalized orthopair fuzzy sets. IEEE Trans Fuzzy Syst 25(5):1222-1230

Ye J, Ai Z, Xu Z (2019) Single variable differential calculus under q-rung orthopair fuzzy environment: limit, derivative, chain rules, and its application. Int J Intell Syst 34(7):1387-1415

Yu L, Lai KK (2011) A distance-based group decision-making methodology for multi-person multi-criteria emergency decision support. Decision Support Syst 51(2):307-315

Yu F, Du L, Ojcius DM, Pan C, Jiang S (2020) Measures for diagnosing and treating infections by a novel coronavirus responsible for a pneumonia outbreak originating in Wuhan, China. Microbes Infect 22(2):74-9

Zadeh LA (1965) Fuzzy sets. Inf Control 8(3):338-1150

Zeng S (2017) Pythagorean fuzzy multiattribute group decision making with probabilistic information and OWA approach. Int J Intell Syst 32(11):1136-1150

Zhang H (2014) Linguistic intuitionistic fuzzy sets and application in MAGDM. J Appl Math. https://doi.org/10.1155/2014/432092

Publisher's Note Springer Nature remains neutral with regard to jurisdictional claims in published maps and institutional affiliations. 